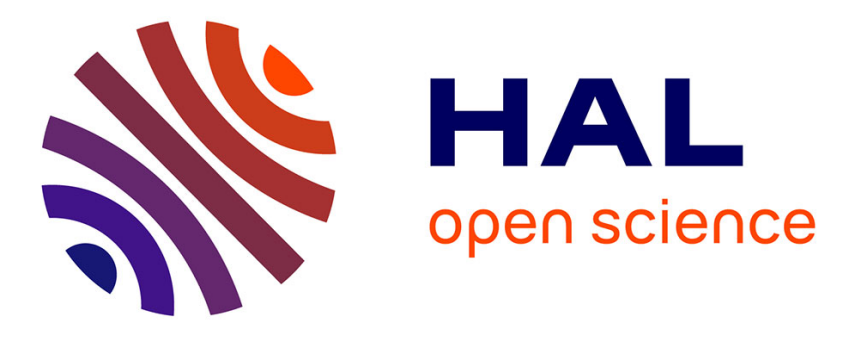

\title{
Traitement STAP et Modélisation SIRV : Robustesse et Persymétrie
}

Jean-Philippe Ovarlez, Frédéric Pascal, Philippe Forster, Guillaume Ginolhac, Mélanie Mahot

\section{- To cite this version:}

Jean-Philippe Ovarlez, Frédéric Pascal, Philippe Forster, Guillaume Ginolhac, Mélanie Mahot. Traitement STAP et Modélisation SIRV : Robustesse et Persymétrie. Traitement du Signal, 2011, 28 (1-2), pp.113-142. 10.3166/ts.28.113-142 . hal-00659845

\section{HAL Id: hal-00659845 \\ https://hal-centralesupelec.archives-ouvertes.fr/hal-00659845}

Submitted on 4 Mar 2020

HAL is a multi-disciplinary open access archive for the deposit and dissemination of scientific research documents, whether they are published or not. The documents may come from teaching and research institutions in France or abroad, or from public or private research centers.
L'archive ouverte pluridisciplinaire HAL, est destinée au dépôt et à la diffusion de documents scientifiques de niveau recherche, publiés ou non, émanant des établissements d'enseignement et de recherche français ou étrangers, des laboratoires publics ou privés. 


\title{
Traitement STAP et ModÈlisation SIRV : Robustesse et PersymÈtrie
}

\author{
Jean-Philippe Ovarlez ${ }^{* * * * *}$ - Frédéric Pascal $^{* * *}$ - Philippe \\ Forster $^{* *}$ - Guillaume Ginolhac ${ }^{* *}$ - MÈlanie Mahot ${ }^{\text {*** }}$
}

* ONERA, the French Aerospace Lab

DEMR/TSI,

BP 80100

F-91123 Palaiseau Cedex, France

jean-philippe.ovarlez@onera.fr

** ENS Cachan

SATIE, CNRS, UniverSud

61, av Président Wilson

F-94230 Cachan, France

\{philippe.forster,guillaume.ginolhac\}@satie.ens-cachan.fr

*** Supélec, Plateau du Moulon

SONDRA

3 rue Joliot-Curie

F-91192 Gif-sur-Yvette Cedex, France

\{frederic.pascal,melanie.mahot\}@supelec.fr

RÉSUMÉ. Ce papier dÈcrit l'intèrÍt de l'apport des processus SIRV (processus gaussiens compo-

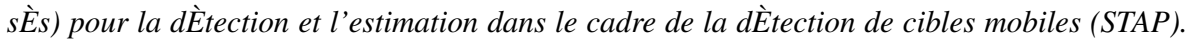
Ces processus permettent de maniËre ÈlÈgante et efficace d'Ètendre au problËme STAP la

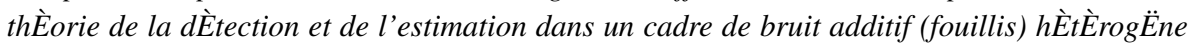
et/ou non gaussien. Nous prÈsentons ainsi les outils thÈoriques (estimateurs, ḋ̀tecteurs) qui permettent d'amÈliorer les performances des dÈtecteurs conventionnels ainsi que leurs pro-

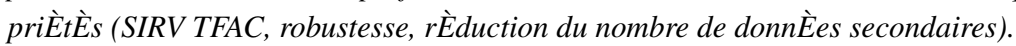

ABSTRACT. In the STAP framework, this paper proposes a review of SIRV modelling for detection and estimation. These processes allow to extend the detection and estimation theory to the STAP problem in a non-Gaussian and heterogeneous background. Some theoretical tools (estimators, detectors) are presented jointly with their attractive properties (SIRV CFAR, robust-

$1^{\text {re }}$ soumission à Traitement du signal, le 1er juillet 2011 
$21^{\text {re }}$ soumission à Traitement du signal

ness, secondary data number reduction) which allow to significantly outperform conventional, e.g. Gaussian based, ones

MOTS-CLÉS : DÈtection adaptative, STAP, fouillis gaussien et non-gaussien, thÈorie des SIRV, estimation de matrice de covariance, persymĖtrie, estimation robuste

KEYWORDS: Adaptive signal detection, STAP, Gaussian and non-Gaussian clutter, SIRV theory, covariance matrix estimation, persymmetry, robust estimation 


\section{Extended Abstract}

Space Time Adaptive Processing (STAP) is a recent technique used in airborne phased array radar to detect moving targets embedded in an interference background such as jamming or strong clutter. While conventional radars are capable of detecting targets both in the time domain related to target range and in the frequency domain related to target velocity, STAP uses an additional domain (space or information collected by an antennas array) related to the target angular localization. The joint processing of these space-time data, by appropriate two-dimensional adaptive filtering methods, allows stronger interference/clutter rejection and therefore improved target detection. Moreover, STAP can now be used in High Resolution (STAP-HR) for a better classification of the moving target. But in this case, the widely used hypothesis of a Gaussian clutter is not valid anymore because of the spatial heterogeneity of the clutter (clutter power, called the texture, may change spatially) and detection performance significantly decreases. For example, in the area of STAP High Resolution (STAP-HR), the resolution is such that the Central Limit Theorem cannot be applied anymore since the number of backscatters is too small. Equivalently, it is known that reflected signals could be very impulsive in low grazing angle radar. This is why, in the last decades, the radar community has been very interested on problems dealing with non-Gaussian clutter modeling.

To fill these gaps, non-Gaussian models for the clutter have to be considered. In the literature of radar detection and estimation, the Spherically Invariant Random Vector (SIRV) modelling is generally used for its good statistical properties and for its good fitting to real data. More precisely, a SIRV is the product of a Gaussian random process with the square root of a non-negative random scalar variable (the so-called texture). Thus, the SIRV is fully characterized by the texture (representing an unknown power) and the unknown covariance matrix of the zero-mean Gaussian vector. One of the major challenging difficulties in SIRV modeling is to estimate these two unknown quantities and particularly the Clutter Covariance Matrix for STAP applications of our concern, better the accuracy of this estimate, better the detection performance. Furthermore, this model provides a very general framework which includes classical distributions as for example the Gaussian distribution, the K-distribution or the Weibull distribution. The Clutter Covariance Matrix is estimated from signal-free and independent data, called the secondary data. Under non-Gaussian clutter assumptions, this involves several difficulties. First, the spatial heterogeneity of the clutter deteriorates the Clutter Covariance Matrix estimation accuracy. Moreover, if the secondary data are not samples of the same parameterized distribution, i.e. the same covariance matrix, this estimation makes no sense: this is the case for example in non-side looking configuration where the Clutter Covariance Matrix is non stationary from cell to cell: this drawback is called frequency heterogeneity clutter. Secondary data may also be corrupted by a high number of targets, interferences or jamming. The conventional techniques based on the empirical estimation of the Clutter Covariance Matrix are therefore not robust. 
We propose in this paper to analyze and to solve, in the SIRV theory framework and more generally in the context of robust statistics, these typical effects (spatial heterogeneity of the clutter intensity, clutter heterogeneity, influence of the number of secondary data, influence of the contaminated secondary date) arising in modern STAP analysis.

In the first part of the paper, we propose to describe the SIRV modelling for clutter background. In this context, all the conventional (Gaussian background) and more recent techniques on detection and parameters estimation in non-Gaussian background are recalled. This leads to define a new kind of detector (Adaptive Normalized Matched Filter) and a new kind of Clutter Covariance Matrix estimation (Fixed Point Covariance Matrix). Jointly combined, they characterize a new STAP detector which has the SIRV-CFAR property. This latter property means that this detector under clutter assumption only does not depend on the texture and on the covariance matrix. It can so be robust when dealing with clutter heterogeneity, clutter transition and so warrants Constant False Alarm Rate. The second part describes the robustness of the proposed detector with respect to targets, jammers, strong echoes present in the contaminated secondary data. The third part will focus on the a priori hypothesis that can be made on the structure of the Clutter Covariance Matrix, i.e., the persymmetry property that characterizes the STAP in side-looking configuration. This property will lead to reduce by a factor two the number of secondary data needed to estimate the covariance matrix and this for the same performance. The latter detector, taking into account this property of persymmetry will so be extended in SIRV context. The last part is devoted to the STAP results obtained on experimental data provided by the DGA/MI. They show that the proposed STAP detector has the same performance in a classical Gaussian background but can significantly improves them in non-Gaussian clutter.

All the presented works are here focused on the STAP application. However, since the theoretical tools are very general, they can be easily extended for other radar applications (SAR images classification, MIMO radar, Polarimetry, hyperspectral data, etc.). 


\section{Introduction}

La dÈtection STAP des cibles mobiles par un capteur aÈroportÈ de surveillance du sol ou par une antenne de pointe avant de chasseur est un sujet de recherche qui suscite actuellement un grand intÈrÍt opÈrationnel (?). À líaide díun rÈseau díantennes, le principe du STAP est díĖliminer conjointement par traitement temporel et spatial les Èchos fixes du sol (fouillis) pouvant considÈrablement dÈtÈriorer les performances de dÈtection díune cible díintÈrÍt (?).

Les schÈmas de dÈtection cohÈrente de cibles (radar de veille, poursuite, STAP, etc.) dans un environnement de fouillis s'appuient gÈnÈralement sur le critËre de

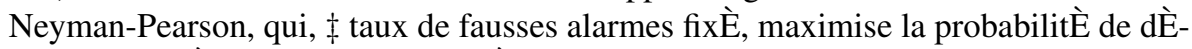
tection. Le dÈtecteur optimal, bas ̇̀ sur la formation du rapport de Vraisemblance, n'est possible $\ddagger$ mettre en úuvre que lorsque la statistique du bruit environnant est connue a priori. Lorsque les paramËtres rËgissant le fouillis (matrice de covariance, paramËtres de la loi a priori le caractĖrisant, ...) ne sont pas connus, il est nÈcessaire de les estimer (gÈnÈralement par Maximum de Vraisemblance) et de construire le dÈtecteur GLRT associè. La qualitÈ díestimation de ces paramËtres influe fortement sur les performances attendues du dÈtecteur.

Le dÈtecteur le plus couramment rencontrÈ est bas ̇̀ sur une statistique gaussienne du bruit ou du fouillis et caractÈrise plus communÈment en radar le filtre adaptÈ aprËs blanchiment. Ce dÈtecteur nÈcessite líestimation de la matrice de covariance des signaux temporels collectÈs sur le rÈseau de capteurs dans une zone spatiale de fouillis situÈe autour de la cible díintÈrÍt (caractÈrisant les donnÈes dites secondaires). Cette matrice de covariance est gÈnÈralement estimÈe empiriquement et appelÈe SCM (Sample Covariance Matrix).

Une analyse bibliographique montre toutefois que beaucoup d'algorithmes de dÈtection proposÈs sont majoritairement basÈs sur líhypothËse díun fouillis gaussien supposË spatialement homogËne. Cette hypothËse simplificatrice níest plus respectÈe lorsque la zone de fouillis níest plus homogËne, lorsque la cellule díanalyse tend $\ddagger$ devenir de plus en plus petite ou encore lorsque l'Èclairement du fouillis est en incidence rasante. Ceci entraÓne une dÈgradation des performances de rÈjection de fouillis et implicitement de dÈtection (?).

Des travaux assez rÈcents ont permis de dÈfinir de nouveaux types de dÈtecteurs en environnement non gaussien et non homogËne. Ces nouveaux dÈtecteurs sont construits sur líhypothËse trËs gÈnÈrale díun fouillis considÈrÈ comme processus gaussien composÈ, cíest-ł-dire un processus gaussien (speckle) dont la puissance (texture) est elle-mÍme alÈatoire. Ces processus permettent de reprÈsenter une zone de fouillis hÈtÈrogËne spatialement et de prendre en compte des Èventuelles fluctuations spectrales dues au vent par exemple. Ce processus composè, appelÈ SIRP (Spherically Invariant Random Process), possËde un nombre de degrÈs de libertÈ supËrieur au cas Gaussien et englobe de ce fait une infinitÈ de lois statistiques (Weibull, K-distribution, alpha-stable,...). Ces nouveaux dÈtecteurs adaptatifs, bas Ès sur une nouvelle estimation de la matrice de covariance du fouillis (appelÈe matrice du point 
fixe), sont alors invariants $\ddagger$ la texture (variation spatiale de la puissance de fouillis) mais Ègalement invariants aux Èventuelles fluctuations spectrales du fouillis.

Ces nouveaux dÈtecteurs et processus díestimation des matrices de covariance ont ÈtÈ appliquÈs dans le cadre de la dÈtection STAP et apportent une amÈlioration trËs nette des performances de dÈtection par rapport $\ddagger$ celles díun traitement classique. Ils peuvent Ègalement Ítre trËs efficaces dans le cas de la prËsence Èventuelle de nombreuses cibles ou de brouilleurs dans les donnÈes secondaires . Líestimateur classique de la matrice de covariance síavËre alors complËtement biaisÈ par la prËsence de ces cibles ou brouilleurs et dÈgrade fortement les performances attendues du dÈtecteur. La matrice de covariance du point fixe possËde une propriÈtÈ statistique trËs importante : la robustesse. Cette robustesse caractËrise le pouvoir díinvariance $\ddagger$ un Ècart sur les hypothËses faites pour le modËle : ici, les donnÈes secondaires sont polluËes par la prËsence de cibles et síĖcartent de ce fait de líhypothËse díhomogÈnËitÈ sur la densitÈ de probabilitÈ les rÈgissant. MÍme dans le cas conventionnel gaussien, ces estimateurs permettent de s'affranchir des donnÈes secondaires polluËes. Ces techniques ont encore ĖtÈ Ètendues (?) pour rËpondre au problËme crucial de la rÈduction du nombre de cases distance nÈcessaire $\ddagger$ l'estimation de la matrice de covariance du fouillis. Elles s'appuient sur l'information a priori que l'on peut donner $\ddagger$ cette matrice de covariance. En utilisant la propriÈtÈ a priori de persymÈtrie (symÈtrie par rapport $\ddagger$ l'antidiagonale) de cette matrice que l'on rencontre en STAP latÈral conventionnel, il est possible, $\ddagger$ nombre de cases distance donnÈ, d'amÈliorer encore les performances du dËtecteur STAP.

Ce papier est organisÈ comme suit. Dans le paragraphe 2, nous rappelons les techniques utilisÈes dans le cadre de la dÈtection radar (le STAP Ètant un cas particulier) et de l'estimation des paramËtres de fouillis (matrice de covariance) en environnement gaussien et non gaussien. Le paragraphe 3 s'Ėtend sur la propriĖtÈ de robustesse qui caractËrise ces estimateurs. Dans le paragraphe 4, nous Ètendons les prÈcÈdentes techniques $\ddagger$ la prise en compte d'une information a priori sur la structure de la matrice de covariance du fouillis, la persymÈtrie, que l'on rencontre dans le cadre du STAP latÈral et qui permet de proposer des estimateurs et des dÈtecteurs plus efficaces. Ces techniques sont enfin ÈvaluÈes dans le paragraphe 5 sur des donnËes issues du club STAP (?).

\section{DÈtection et Estimation pour le STAP}

En dÈtection radar, le principal problËme consiste gÈnÈralement $\ddagger$ dÈtecter, dans un vecteur díobservation $\boldsymbol{x}$ de dimension $m$ (correspondant par exemple en STAP $\ddagger$ la concatÈnation des $M$ impulsions Èmises par $N$ antennes, $m=M N$ ), un vecteur $\alpha \boldsymbol{a}$, d'amplitude complexe $\alpha$ gÈnÈralement inconnue et de steering vector $\boldsymbol{a}$ caractÈrisant les paramËtres $\boldsymbol{\theta}$ inconnus de la cible (position, vitesse, rÈponse polarimÈtrique, direction, etc.) corrompu par un bruit additif de fouillis $\boldsymbol{c}$ de densitË $p_{\boldsymbol{c}}($. et caractËrisÈ par un vecteur $\boldsymbol{\theta}_{\boldsymbol{c}}$ de paramËtres inconnus (moyenne, variance, cova- 
riance, paramËtres de forme, etc.) . Ce problËme se formalise gÈnÈralement par un test díhypothËses binaires :

$$
\left\{\begin{array}{lll}
H_{0}: \boldsymbol{x}=\boldsymbol{c} & \boldsymbol{x}_{i}=\boldsymbol{c}_{i} & i=1, \ldots, K \\
H_{1}: \boldsymbol{x}=\alpha \boldsymbol{a}+\boldsymbol{c} & \boldsymbol{x}_{i}=\boldsymbol{c}_{i} & i=1, \ldots, K
\end{array}\right.
$$

o les $\boldsymbol{x}_{i}$ sont les $K$ vecteurs díobservations indÈpendants appelÈs donnt̀es secondaires, contenant uniquement le bruit de fouillis et permettant díestimer les paramËtres du fouillis (matrice de covariance, puissance, densitÈ, ...), puis $\ddagger$ terme de construire un dÈtecteur optimal.

Dans le cas de paramËtres $\boldsymbol{\theta}$ et $\boldsymbol{\theta}_{\boldsymbol{c}}$ connus, le dÈtecteur optimal, construit selon la thÈorie de Neyman Pearson, est basĚ sur le test du rapport de vraisemblance :

$$
\Lambda(\boldsymbol{x})=\frac{p_{\boldsymbol{c}}(\boldsymbol{x}-\alpha \boldsymbol{a})}{p_{\boldsymbol{c}}(\boldsymbol{x} ; \boldsymbol{\theta})} .
$$

Dans le cas de paramËtres de cibles ou de fouillis inconnus, le dÈtecteur optimal est le dÈtecteur construit par le rapport de vraisemblance gÈnÈralisÈ (GLRT) :

$$
\Lambda(\boldsymbol{x})=\frac{\max _{\alpha, \boldsymbol{\theta}, \boldsymbol{\theta}_{\boldsymbol{c}}} p_{\boldsymbol{c}}(\boldsymbol{x}-\alpha \boldsymbol{a})}{\max _{\boldsymbol{\theta}_{\boldsymbol{c}}} p_{\boldsymbol{c}}(\boldsymbol{x})} .
$$

Cette expression est souvent difficile $\ddagger$ obtenir explicitement. Une alternative consiste $\ddagger$ estimer les paramËtres de fouillis directement $\ddagger$ l'aide de donnÈes secondaires (ne contenant pas la cible) et de rÈinjecter ces estimÈes dans le rapport de vraisemblance (two steps GLRT). Dans le cas gaussien, le dÈtecteur adaptatif de (?; ?) est un vrai dÈtecteur GLRT.

\subsection{Fouillis gaussien}

Dans le contexte statistique gaussien, $\boldsymbol{c}$ et $\left(\boldsymbol{c}_{k}\right)_{k \in[1, N]}$ sont des vecteurs complexes gaussiens $\mathcal{C N}_{m}(\mathbf{0}, \boldsymbol{M})$, centrÈs, circulaires, indÈpendants deux $\ddagger$ deux, de mÍme matrice de covariance $\boldsymbol{M}$, c'est $\ddagger$ dire distribuÈs selon la loi :

$$
p_{\boldsymbol{c}}(\boldsymbol{c})=\frac{1}{\pi^{m}|\boldsymbol{M}|} \exp \left(-\boldsymbol{c}^{H} \boldsymbol{M}^{-1} \boldsymbol{c}\right) .
$$

Lorsque $M$ est connue et l'amplitude $\alpha$ de la cible inconnue, le test du rapport de vraisemblance gÈnÈralisÈ est connu sous la dÈnomination cÈlËbre du test du filtre adaptÈ (Matched Filter) :

$$
\Lambda_{M F}(\boldsymbol{M}, \boldsymbol{x})=\frac{\left|\boldsymbol{a}^{H} \boldsymbol{M}^{-1} \boldsymbol{x}\right|^{2}}{\boldsymbol{a}^{H} \boldsymbol{M}^{-1} \boldsymbol{a}} \underset{H_{0}}{\gtrless} \lambda_{M F},
$$


o $\lambda_{M F}$, le seuil de dÈtection, est reliè $\ddagger$ la PFA $P_{f a}$ par la relation :

$$
\lambda_{M F}=-\ln P_{f a} .
$$

Notons que la loi du test ne dÈpend pas de la matrice $M$ : ce dÈtecteur est donc $\ddagger$ Taux de Fausse Alarme Constant (CFAR) vis $\ddagger$ vis du fouillis.

En pratique, cette matrice $M$ n'est pourtant pas connue explicitement et il est nÈcessaire de l'estimer. Dans ce cadre, les tests de dÈtection rËpondant $\ddagger$ ce besoin sont dits adaptatifs. Les premiers travaux dans ce domaine sont dus $\ddagger$ (?; ?), suivis par les travaux de (?; ?; ?; ?). Une solution simple, consiste par exemple, $\ddagger$ substituer $\boldsymbol{M}$ dans [??] par un estimateur $\widehat{\boldsymbol{M}}$ construit sur les donnÈes secondaires $\left(\boldsymbol{c}_{k}\right)_{k \in[1, K]}$. Lorsqu' aucune information a priori n'est connue sur la structure de $M$, la matrice de covariance empirique (SCM), estimateur solution du maximum de vraisemblance, est trËs largement utilisÈe :

$$
\widehat{\boldsymbol{M}}_{S C M}=\frac{1}{K} \sum_{k=1}^{K} \boldsymbol{x}_{k} \boldsymbol{x}_{k}^{H},
$$

et conduit $\ddagger$ dÈfinir le test de l’Adaptive Matched Filter (AMF) :

$$
\Lambda_{A M F}\left(\widehat{\boldsymbol{M}}_{S C M}, \boldsymbol{x}\right)=\frac{\left|\boldsymbol{a}^{H} \widehat{\boldsymbol{M}}_{S C M}^{-1} \boldsymbol{x}\right|^{2}}{\boldsymbol{a}^{H} \widehat{\boldsymbol{M}}_{S C M}^{-1} \boldsymbol{a}}{\underset{H}{H_{0}}}_{H_{1}}^{\lambda_{A M F}} .
$$

L'AMF est un two-step GLRT. La relation analytique liant la PFA $P_{f a}$ et le seuil de dÈtection $\lambda_{A M F}$ est ainsi donnÈe par (?; ?) :

$$
P_{f a}={ }_{2} F_{1}\left(K-m+1, K-m+2 ; K+1 ;-\frac{\lambda_{A M F}}{K}\right),
$$

$\mathrm{O}_{2} F_{1}($.$) est la fonction hypergÈomÈtrique (?) dÈfinie par$

$$
{ }_{2} F_{1}(a, b ; c ; z)=\frac{\Gamma(c)}{\Gamma(b) \Gamma(c-b)} \int_{0}^{1} \frac{t^{b-1}(1-t)^{c-b-1}}{(1-t z)^{a}} d t .
$$

Cette expression montre que la valeur du seuil de dÈtection assurant une PFA donnÈe est indËpendante de la matrice de covariance $M$ du fouillis : líAMF est CFAR par rapport $\ddagger \boldsymbol{M}$. Si le fouillis n’est plus homogËne (cas des transitions de fouillis) ou devient non-gaussien, ce dÈtecteur ne garantit plus une bonne rÈgulation de la fausse alarme (rÈglage du seuil) qui peut varier selon la nature du fouillis rencontrÈ.

\subsection{Fouillis non-gaussien}

De nombreux travaux (?; ?) ont montrÈ que la statistique des donnÈes de fouillis collectÈes par un radar s'Èloignait de la statistique gaussienne. De nombreux papiers 
ont ainsi tentÈ de caractÈriser la densitÈ de probabilitÈ de ce fouillis par des lois de Weibull, les lois K-distributions, les loi gamma, ... En fait, toutes ces lois peuvent Ítre, de maniËre ĖlÈgante, englobÈes dans une immense famille de processus appelÈe SIRP (Spherically Invariant Random Process). Ces processus (?; ?; ?; ?), Ègalement appelĖs, gaussiens composÈs, sont des processus alËatoires gaussiens dont la variance est Ègalement alÈatoire. On comprend alors immÈdiatement l'intÈrÍt de ces processus pour la modÈlisation du fouillis radar. Le fouillis radar provient de la sommation amplitude-phase des diffĖrents rÈflecteurs du sol prÈsents dans la cellule analysÈe. De par la variabilitÈ de l'environnement, l'angle de vue de la cellule analysĖe, les rÈflecteurs changent, d'une cellule $\ddagger$ l'autre, d'orientation, de nature, de rËflectivitÈ et varient en nombre. La puissance du signal rËtrodiffusè par la cellule analysĖe devient alors alÈatoire. C'est cet aspect alÈatoire qui rend complexe l'analyse du fouillis.

\subsubsection{Processus SIRP et Vecteurs SIRV}

Les modËles SIRP permettent de prendre en compte de maniËre gÈnÈrale et ÈlÈgante cet aspect alÈatoire par un double processus alÈatoire : un processus gaussien appelÈ speckle †dont la puissance, appelÈe texture est elle-mÍme alÈatoire. L'exemple de la K-distribution, processus SIRP dont la puissance est gamma-distribuÈe, illustre en quelque sorte la connexion du modËle avec la physique du radar : si la case d'analyse contient, de par sa taille, une infinitÈ de rÈflecteurs supposÈs identiquement distribuĖs, le ThËorËme Central Limite nous assure un comportement statistique de type gaussien. Si la distribution du nombre de rÈflecteurs est une loi binÙmiale nÈgative, l’Ècho rËtrodiffusÈ est K-distribuÈ.

Il est possible d'Ètendre la thÈorie des SIRP au cas multivariÈ (?). La thÈorie nous conduit alors $\ddagger$ la dÈfinition des SIRV (Spherically Invariant Random Vector). Plus prÈcisÈment, un SIRV $c$ est le produit de la racine carrÈe d'une variable alËatoire scalaire positive $\tau$, appelÈe la texture et caractĖrisÈe par sa densitÈ de probabilitÈ $p_{\tau}($.$) et d'un vecteur alÈatoire complexe \boldsymbol{g}$, appelÈ speckle, de dimension $m$, gaussien, centrè, circulaire et ayant pour matrice de covariance $\boldsymbol{M}=\mathcal{E}\left\{\boldsymbol{g} \boldsymbol{g}^{H}\right\}$ telle que $\operatorname{tr}\{\boldsymbol{M}\}=m$ pour des considÈrations d'identifiabilitÈ et o $\mathcal{E}\{$.$\} dÈsigne l'espÈ-$ rance mathÈmatique d'une variable alÈatoire :

$$
\boldsymbol{c}=\sqrt{\tau} \boldsymbol{g} .
$$

D'autre part, la densitÈ de probabilitÈ (PDF) $p_{c}($.$) du vecteur c$ est connue et son expression est la suivante :

$$
p_{\boldsymbol{c}}(\boldsymbol{c})=\int_{0}^{+\infty} \frac{1}{(\pi \tau)^{m}|\boldsymbol{M}|} \exp \left(-\frac{\boldsymbol{c}^{H} \boldsymbol{M}^{-1} \boldsymbol{c}}{\tau}\right) p_{\tau}(\tau) d \tau .
$$

Conditionnellement $\ddagger$ la case d'analyse ou $\ddagger \tau$, le vecteur $c$ de donnÈes collectÈes est supposè gaussien et la matrice de covariance $\boldsymbol{M}$ gËre ainsi le degrÈ de corrÈlation sur ce vecteur. De case d'analyse $\ddagger$ case d'analyse, la texture $\tau$ modÈlise le fait que la norme du vecteur d'observation $c$ varie alÈatoirement. On peut comprendre l'interprËtation physique de cette matrice et de la texture dans les cas diffÈrents suivants : 
- pour un radar Èmettant pÈriodiquement des impulsions et dÈcoupant l'axe distance en cases de rËsolution, la matrice de covariance, gËre, dans une case distance donnÈe, la corrÈlation existant entre les diffÈrentes impulsions. Cette information de corrÈlation est reliÈe au caractËre spectral (ou Doppler) du fouillis (vent sur une forĺt, fouillis dynamique de la mer) et influe sur les performances de dËtection. La texture gËre ici la variation de la norme du vecteur sur l'axe des distances,

- pour un rÈseau de capteurs, la matrice de corrÈlation gËre la corrÈlation existant sur l'axe angulaire. La texture peut reprÈsenter la variation temporelle de la puissance,

- pour un traitement de type STAP, la matrice de covariance gËre $\ddagger$ la fois l'aspect spectral et angulaire du fouillis, la texture pilotant plutÙt la variation de puissance des Èchos collectÈs sur l'axe distance,

- pour une image SAR polarimĖtrique, interfÈromÈtrique voire les deux, la matrice de covariance (dite de cohÈrence), calculÈe sur le domaine spatial des pixels de l'image SAR, gËre la corrÈlation existant sur ces diffËrents canaux (?). La texture reprËsente dans ce cas, la variation spatiale de la puissance de vecteurs collectÈs de pixel $\ddagger$ pixel.

Une des nombreuses propriÈtÈs des SIRV est l'invariance de la loi de la texture au filtrage (filtre linĖaire invariant dans le temps). AprËs passage dans un filtre caractÈrisè par sa matrice de transformation $\boldsymbol{A}$, le SIRV $\boldsymbol{c}$ reste un SIRV de mÍme loi $p_{\tau}($. mais sa matrice de covariance Èvolue comme dans le cas gaussien, soit $\boldsymbol{A} \boldsymbol{M} \boldsymbol{A}^{H}$. Pour le traitement radar, qui, gÈnÈralement, commence par comprimer ses impulsions reÁus (filtrage adaptÈ distance) sur l'axe des distances ( $\boldsymbol{A}$ caractÈrise alors le code Èmis), cette opÈration ne change pas la nature statistique du fouillis mais seulement son contenu spectral.

Cette modÈlisation des processus SIRV est donc trËs gÈnÈrale et peut s'adapter $\ddagger$ de nombreux problËmes radar ou autres. L'intĖrít de cette modÈlisation est qu'elle englobe Ègalement les processus gaussiens, ce qui la rend encore plus intÈressante

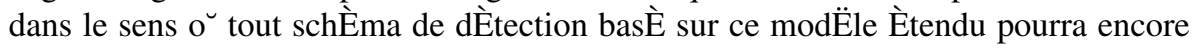
s'appliquer dans le cas classique, c'est $\ddagger$ dire s'adapter au cas gaussien.

\subsubsection{DÈtection dans les SIRV}

Pour de nombreux modËles de texture du processus SIRV, on peut dÈterminer le dÈtecteur optimal associè (rapport de formes intËgrales). Cependant, la forme du rapport de vraisemblance donnÈ en [??] et associÈ au test de dËtection peut rarement s'expliciter sous forme analytique ce qui le rend difficile $\ddagger$ mettre en œuvre (nÈcessitÈ de connaÓtre la loi de la texture $p_{\tau}($.$) . Lorsque la nature du fouillis change (en$ caractÈristiques, en loi, ...), il est Ègalement nÈcessaire de changer de dÈtecteur ou d'estimer $\ddagger$ nouveau les paramËtres de formes de la loi rÈgissant le fouillis, ce qui est trËs contraignant pour le radariste. Plusieurs travaux ont nËanmoins permis d'Ëtablir de diffÈrentes maniËres, une mÍme forme de dËtecteur trËs intÈressant :

- le NMF (Normalized Matched Filter) proposÈ par (?; ?) : son problËme Ètait, dans le contexte gaussien, de dÈterminer un dÈtecteur invariant au paramËtre 
d'Èchelle (la variance inconnue). Dans le cas des SIRV, ce paramËtre d'Èchelle est ici de maniËre logique la texture. AntÈrieurement, (?; ?) avaient dÈjł obtenu cette expression dans des hypothËses de bruit Gaussien,

- le GLRT-LQ (Generalized Likelihood Ratio Test ñ Linear Quadratic) dÈfini par (?; ?). Les dĖtecteurs optimaux basÈs sur les processus SIRV Ėtant dĖfinis par des formes intÈgrales et dÈpendantes de la densitÈ de probabilitÈ de la texture, le GLRTLQ est dÈfini comme approximation asymptotique du dÈtecteur optimal dans un environnement de bruit K-distribuÈ,

- (?) líobtiennent en considÈrant la texture du SIRV comme un paramËtre dÈterministe et en la remplaÁant par son estimÈe au sens du Maximum de Vraisemblance sous chacune des hypothËses,

- le BORD (Bayesian Optimum Radar Detector) asymptotique dÈfini par (?) est obtenu par modÈlisation bayĖsienne de la loi de la texture $p_{\tau}($.$) et en faisant tendre$ le nombre $K$ de donnÈes secondaires vers l'infini.

Lorsque la matrice de covariance $M$ du speckle est connue, tous les travaux citÈs prÈcÈdemment convergent vers une forme totalement Èquivalente du test de dÈtection, conduisant au NMF (Normalized Matched Filter) :

$$
\Lambda_{N M F}(\boldsymbol{M}, \boldsymbol{x})=\frac{\left|\boldsymbol{a}^{H} \boldsymbol{M}^{-1} \boldsymbol{x}\right|^{2}}{\left(\boldsymbol{a}^{H} \boldsymbol{M}^{-1} \boldsymbol{a}\right)\left(\boldsymbol{x}^{H} \boldsymbol{M}^{-1} \boldsymbol{x}\right)}{\underset{H}{H_{0}}}_{H_{1}}^{H_{N M F}},
$$

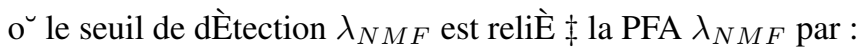

$$
\lambda_{N M F}=1-P_{f a}^{\frac{-1}{1-m}} .
$$

Ce dÈtecteur est homogËne pour les variables $x, M$ et $\boldsymbol{a}:$ n'importe quel changement d'Èchelle sur ces variables ne change pas la quantitÈ. On peut Ègalement remarquer que [??] est exactement le cosinus carrË de l'angle entre le vecteur de test $\boldsymbol{x}$ et le steering vector $\boldsymbol{a}$. Ce dÈtecteur est quelquefois appelÈ dÈtecteur d'angle contrairement au dËtecteur MF donnÈ en [??] qui est plutÙt un dÈtecteur de puissance. Ce dÈtecteur est connu pour Ítre TFAC vis $\ddagger$ vis de la texture inconnue $\tau$ et de la matrice de covariance $\boldsymbol{M}$, ce qui permet de parfaitement rËguler son taux de fausses alarmes (voir figure ??). On dit qu'il est SIRV-TFAC. Cette propriÈtÈ est trËs recherchÈe en environnement hÈtĖrogËne, dans les transitions de fouillis car ce dÈtecteur s'affranchit ainsi de la variation de puissance (la texture) de case de test $\ddagger$ case de test.

Lorsque $M$ est inconnue, une solution trËs simple (procÈdure qui n'est pas exactement un GLRT) consiste $\ddagger$ remplacer la matrice de covariance $M$ par une estimÈe $\widehat{\boldsymbol{M}}$ dans [??]. Cet estimateur, notÈ $\widehat{\boldsymbol{M}}$, est alors construit sur les donnÈes secondaires $\left(\boldsymbol{c}_{k}\right)_{k \in[1, N]}$ qui sont supposĖes ne contenir que le fouillis. Ce nouveau dÈtecteur (?; ?) 


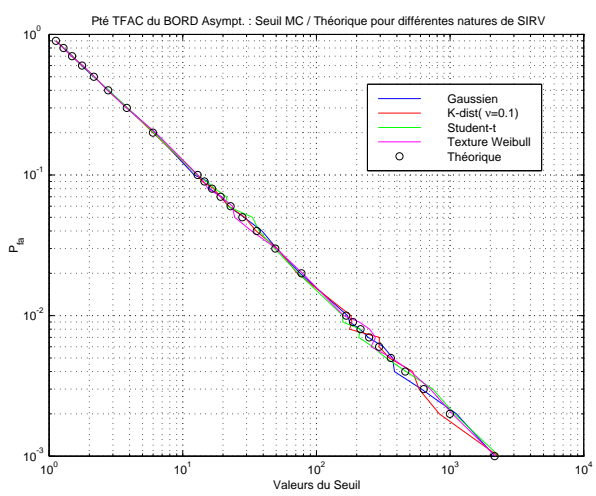

Figure 1. Propriṫtè TFAC (CFAR) du BORD asymptotique ou du NMF

est appelÈ Adaptive Coherence Estimate (ACE) ou encore Adaptive Normalized Matched Filter (ANMF) et prend la forme :

$$
\Lambda_{A N M F}(\widehat{\boldsymbol{M}}, \boldsymbol{x})=\frac{\left|\boldsymbol{a}^{H} \widehat{\boldsymbol{M}}^{-1} \boldsymbol{x}\right|^{2}}{\left(\boldsymbol{a}^{H} \widehat{\boldsymbol{M}}^{-1} \boldsymbol{a}\right)\left(\boldsymbol{x}^{H} \widehat{\boldsymbol{M}}^{-1} \boldsymbol{x}\right)} \underset{H_{0}}{\mathrm{H}_{1}} \lambda_{A N M F} .
$$

\subsubsection{Estimation dans les processus SIRV}

Bien entendu, le choix de l'estimateur $\widehat{M}$ est ici primordial. Si l'on souhaite, par exemple, conserver la propriÈtÈ texture TFAC du dËtecteur donnÈ en [??], il est nÈcessaire de trouver un estimateur de matrice de covariance invariant lui aussi $\ddagger$ cette texture.

Le premier type d'estimateur de la matrice de covariance que nous avons rencontrÈ est Èvidemment l'estimateur empirique $\widehat{\boldsymbol{M}}=\widehat{\boldsymbol{M}}_{S C M}$ de la covariance (Sample Covariance Matrix) construit $\ddagger$ partir de $K m$-vecteurs $\boldsymbol{c}_{k}$ de donnÈes secondaires :

$$
\widehat{\boldsymbol{M}}_{S C M}=\frac{1}{K} \sum_{k=1}^{K} \boldsymbol{c}_{k} \boldsymbol{c}_{k}^{H} .
$$

Cet estimateur, la solution du Maximum de Vraisemblance du cas gaussien, distribuÈ selon la loi de Wishart, n'est pourtant pas adaptÈ au cas gÈnÈral des SIRV car sa construction fait intervenir l'information de texture qui biaise l'estimateur. En effet, en remplaÁant $\boldsymbol{c}_{k} \operatorname{par} \sqrt{\tau_{k}} \boldsymbol{g}_{k}$, on obtient :

$$
\widehat{\boldsymbol{M}}_{S C M}=\frac{1}{K} \sum_{k=1}^{K} \tau_{k} \boldsymbol{g}_{k} \boldsymbol{g}_{k}^{H} .
$$


L'ANMF construit avec cet estimateur n'est donc pas texture-TFAC puisque la SCM est dÈpendante de la texture. Une solution pour s'affranchir de l'information de texture a ÈtÈ dÈfinie par Conte et Gini : un nouvel estimateur, la NSCM (Normalized Sample Covariance Matrix), construit sur l'estimateur empirique des donnÈes normalisËes par leur puissance, s'est avÈrÈ invariant $\ddagger$ cette texture :

$$
\widehat{\boldsymbol{M}}_{N S C M}=\frac{m}{K} \sum_{k=1}^{K} \frac{\boldsymbol{c}_{k} \boldsymbol{c}_{k}^{H}}{\boldsymbol{c}_{k}^{H} \boldsymbol{c}_{k}}=\frac{m}{K} \sum_{k=1}^{K} \frac{\boldsymbol{g}_{k} \boldsymbol{g}_{k}^{H}}{\boldsymbol{g}_{k}^{H} \boldsymbol{g}_{k}}
$$

Le dÈtecteur $\Lambda_{A N M F}\left(\boldsymbol{x}, \widehat{\boldsymbol{M}}_{N S C M}\right)$ est donc texture-TFAC. Malheureusement, il n'est pas matrice-TFAC car l'estimateur NSCM est biaisÈ et non consistant (?).

La loi de la matrice de covariance NSCM estimÈe Ètant non connue $\ddagger K$ fixÈ, la loi de l'ANMF donnÈe par [??] n'Ėtait pas si simple $\ddagger$ Ėtablir. L'idÈe de (?) fut de remarquer que, puisque le dÈtecteur Ètait texture-TFAC, une solution Ètait de se placer dans

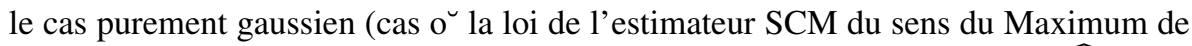
Vraisemblance est distribuÈe selon la loi de Wishart). La loi de $\Lambda_{A N M F}\left(\boldsymbol{x}, \widehat{\boldsymbol{M}}_{S C M}\right)$ conduisant $\ddagger$ l'expression de la relation entre le seuil de dÈtection $\lambda$ et la probabilitE de fausse alarme $P_{f a}$ a pu alors Ítre Ètablie :

$$
P_{f a}=(1-\lambda)^{a-1}{ }_{2} F_{1}(a, a-1 ; b-1 ; \lambda),
$$

avec $a=K-m+2$ et $b=K+2$ et $\mathrm{o}_{2} F_{1}($.$) est la fonction hypergÈomÈtrique.$

Le dÈtecteur $\Lambda_{A N M F}\left(\boldsymbol{x}, \widehat{\boldsymbol{M}}_{S C M}\right)$ Ètait donc matrice-TFAC mais pas textureTFAC.

Nos recherches se sont alors axÈes sur un autre type d'estimateur proposÈ dans la littÈrature : l'estimateur $\widehat{\boldsymbol{M}}=\widehat{\boldsymbol{M}}_{F P}$ du point fixe :

$$
\widehat{\boldsymbol{M}}_{F P}=\frac{m}{K} \sum_{k=1}^{K} \frac{\boldsymbol{c}_{k} \boldsymbol{c}_{k}^{H}}{\boldsymbol{c}_{k}^{H} \widehat{\boldsymbol{M}}_{F P}^{-1} \boldsymbol{c}_{k}} .
$$

Cet estimateur, solution d'une Èquation implicite au point fixe, a ÈtÈ proposÈ par Conte et Gini. Notons que cet estimateur est invariant par changement d'Èchelle des donnÈes $\boldsymbol{c}_{k}$. Ainsi, quelle que soit la puissance de ces donnÈes (texture) rÈgissant les donnÈes secondaires, l'estimateur reste invariant et ne s'occupe que du speckle. Comme pour le NMF, il peut s'obtenir Ègalement de diffÈrentes faÁons :

\section{- AmÈlioration empirique de l'approche NSCM}

Connaissant $\boldsymbol{M}$, la meilleure estimation de la puissance n'est pas $\boldsymbol{c}_{k}^{H} \boldsymbol{c}_{k}$ mais $\boldsymbol{c}_{k}^{H} \boldsymbol{M}^{-1} \boldsymbol{c}_{k}$. L'estimateur empirique des donnÈes normalisÈes par cette nouvelle estimÈe de puissance nous conduit alors $\ddagger 1$ 'estimateur du Point Fixe [??].

\section{- Cas gÈnÈral de la texture alÈatoire}


Dans le cas gÈnÈral des SIRV, on peut dÈterminer l'estimateur $\boldsymbol{M}_{M V}$ du Maximum de Vraisemblance de la matrice $\boldsymbol{M}$. Pour estimer la matrice de covariance $\boldsymbol{M}$ inconnue du processus SIRV, la fonction de vraisemblance des $K$ vecteurs $\boldsymbol{c}_{i}$ supposÈs indÈpendants $\ddagger$ maximiser est donnÈe par :

$$
p_{C}\left(\boldsymbol{c}_{1}, \ldots, \boldsymbol{c}_{K} ; \boldsymbol{M}\right)=\prod_{i=1}^{K} \int_{0}^{+\infty} \frac{1}{\left(\pi \tau_{i}\right)^{m}|\boldsymbol{M}|} \exp \left(-\frac{\boldsymbol{c}_{i}^{H} \boldsymbol{M}^{-1} \boldsymbol{c}_{i}}{\tau_{i}}\right) p_{\tau}\left(\tau_{i}\right) \mathrm{d} \tau_{i} .
$$

En annulant le gradient de [??] par rapport $\ddagger M$, (?) ont ainsi obtenu la solution du Maximum de Vraisemblance suivante :

$$
\begin{aligned}
\widehat{\boldsymbol{M}}_{M V} & =\frac{1}{K} \sum_{i=1}^{K} \frac{h_{m+1}\left(\boldsymbol{c}_{i}^{H} \widehat{\boldsymbol{M}}_{M V}^{-1} \boldsymbol{c}_{i}\right)}{h_{m}\left(\boldsymbol{c}_{i}^{H} \widehat{\boldsymbol{M}}_{M V}^{-1} \boldsymbol{c}_{i}\right)} \boldsymbol{c}_{i} \boldsymbol{c}_{i}^{H}=\frac{1}{K} \sum_{i=1}^{K} c_{m}\left(\boldsymbol{c}_{i}^{H} \widehat{\boldsymbol{M}}_{M V}^{-1} \boldsymbol{c}_{i}\right) \boldsymbol{c}_{i} \boldsymbol{c}_{i}^{H} \\
\mathrm{o} h_{m}(q) & =\int_{0}^{+\infty} \tau^{-m} \exp (-q / \tau) p_{\tau}(\tau) \mathrm{d} \tau \text { et } c_{m}(q)=\frac{h_{m+1}(q)}{h_{m}(q)} .
\end{aligned}
$$

AprËs plusieurs approximations, (?) a obtenu un Maximum de Vraisemblance ApprochÈ $\widehat{\boldsymbol{M}}$ (AML pour Approximate Maximum Likelihood) solution de l'Èquation au point fixe $\widehat{\boldsymbol{M}}=f(\widehat{\boldsymbol{M}})$ avec $f$ dÈfinie par :

$$
f(\widehat{\boldsymbol{M}})=\frac{m}{K} \sum_{i=1}^{K} \frac{\boldsymbol{c}_{i} \boldsymbol{c}_{i}^{H}}{\boldsymbol{c}_{i}^{H} \widehat{\boldsymbol{M}}^{-1} \boldsymbol{c}_{i}} .
$$

Le choix particulier $c_{m}(x)=m / x$ qui correspond en fait $\ddagger$ utiliser le prior impropre de Jeffrey (?) comme densitè de la texture $p_{\tau}($.$) , conduit exactement \ddagger$ l'AML, solution de l'Èquation [??]. Cet AML a l'avantage de ne plus dĖpendre de $h_{m}$ qui dÈpend de la loi de la texture.

\section{- Cas de la texture dÈterministe}

En supposant que le paramËtre $\tau$ est dÈterministe inconnu, la solution du Maximum de Vraisemblance GÈnÈralisÈe pour l'estimation de $M$ est exactement donnÈe l'estimateur du Point Fixe [??]. Cette approche trËs gÈnÈrale a ĖtÈ dÈveloppÈe dans (?) o ${ }^{\smile}$ les $\tau_{i}$ sont supposÈs Ítre des paramËtres inconnus. Conditionnellement aux $\tau_{i}$, la densitÈ des donnÈes est gaussienne. Pour des vecteurs $\boldsymbol{c}_{i}$ indÈpendants, la fonction de vraisemblance correspondante $\ddagger$ maximiser en $\boldsymbol{M}$ et en $\tau_{i}$ est donc donnÈe par :

$$
p_{C}\left(\boldsymbol{c}_{1}, \ldots, \boldsymbol{c}_{K} ; \boldsymbol{M}, \tau_{1}, \ldots, \tau_{K}\right)=\frac{1}{(\pi)^{m K}|\boldsymbol{M}|^{K}} \prod_{i=1}^{K} \frac{1}{\tau_{i}^{m}} \exp \left(-\frac{\boldsymbol{c}_{i}^{H} \boldsymbol{M}^{-1} \boldsymbol{c}_{i}}{\tau_{i}}\right) .
$$

Maximiser cette fonctionnelle en $\tau_{i}$, pour un $M$ fixÈ (mais inconnu), conduit $\ddagger$ :

$$
\hat{\tau}_{i}=\frac{\boldsymbol{c}_{i}^{H} \boldsymbol{M}^{-1} \boldsymbol{c}_{i}}{m} .
$$


En remplaÁant les $\tau_{i}$ dans [??] par leurs estimateurs du Maximum de Vraisemblance $\hat{\tau}_{i}$ dÈfinis par [??], la fonction de vraisemblance rÈduite est donnÈe par :

$$
\hat{p}_{C}\left(\boldsymbol{c}_{1}, \ldots, \boldsymbol{c}_{K} ; \boldsymbol{M}\right)=\frac{1}{(\pi)^{m K}|\boldsymbol{M}|^{K}} \prod_{i=1}^{K} \frac{m^{m} \exp (-m)}{\left(\boldsymbol{c}_{i}^{H} \boldsymbol{M}^{-1} \boldsymbol{c}_{i}\right)^{m}} .
$$

De ce fait, maximiser $\hat{p}_{C}\left(\boldsymbol{c}_{1}, \ldots, \boldsymbol{c}_{K} ; \boldsymbol{M}\right)$ en $\boldsymbol{M}$ revient $\ddagger$ maximiser la fonction suivante $F$ :

$$
F(\boldsymbol{M})=\frac{1}{|\boldsymbol{M}|^{K}} \prod_{i=1}^{K} \frac{1}{\left(\boldsymbol{c}_{i}^{H} \boldsymbol{M}^{-1} \boldsymbol{c}_{i}\right)^{m}} .
$$

L'estimateur du Maximum de Vraisemblance de $M$ est le $\widehat{M}$ qui annule le gradient de $F$, ce qui revient encore $\ddagger$ l'Èquation [??] en $\widehat{\boldsymbol{M}}$ suivante :

$$
\widehat{M}=f(\widehat{M}),
$$

$\mathrm{o}^{\smile}$ la fonction $f($.$) est donnÈe par [??].$

(?) ont montrÈ que l'estimateur du Point Fixe [??], solution d'une Èquation implicite au point fixe, existait, Ètait unique ( $\ddagger$ un facteur d’Èchelle prËs) et pouvait Ítre trËs facilement atteinte par une approche rËcursive du type

$$
\boldsymbol{M}_{n+1}=\frac{m}{K} \sum_{k=1}^{K} \frac{\boldsymbol{c}_{k} \boldsymbol{c}_{k}^{H}}{\boldsymbol{c}_{k}^{H} \boldsymbol{M}_{n}^{-1} \boldsymbol{c}_{k}},
$$

quels que soient le point de dÈpart $M_{0}$ et $n \geq 0$. Le choix particulier et judicieux $\boldsymbol{M}_{0}=\boldsymbol{I}$, nous donne, $\ddagger$ la premiËre itÈration, $\boldsymbol{M}_{1}=\widehat{\boldsymbol{M}}_{N S C M}$, soit la matrice empirique des donnÈes normalisÈes NSCM rencontrÈe en [??] !

On dÈfinit alors $\widehat{\boldsymbol{M}}_{F P}=\lim _{n \rightarrow \infty} \boldsymbol{M}_{n}$. Cet estimateur possËde de remarquables propriÈtÈs statistiques (Pascal et al., 2006; 2008) :

(1) $\widehat{\boldsymbol{M}}_{F P}$ est un estimateur consistant de $\boldsymbol{M}$,

(2) $\widehat{\boldsymbol{M}}_{F P}$ est un estimateur non biaisè de $\boldsymbol{M}$,

(3) la distribution asymptotique de $\widehat{\boldsymbol{M}}_{F P}$ est gaussienne,

(4) cette distribution est la mÍme que la loi asymptotique de Wishart $\ddagger$ $\left(\frac{m}{m+1}\right) K$ degrÈs de libertÈ.

\subsubsection{Propriṫtès SIRV-TFAC des DÈtecteurs}

Le dÈtecteur $\Lambda_{A N M F}\left(\boldsymbol{x}, \widehat{\boldsymbol{M}}_{F P}\right)$ possËde des propriÈtÈs vraiment trËs intÈressantes (Pascal et al., 2004; 2005; 2008). La premiËre est la propriÈtÈ textureTFAC illustrÈe $\ddagger$ la figure ?? qui confirme l'invariance du dÈtecteur $\ddagger$ la texture. La 

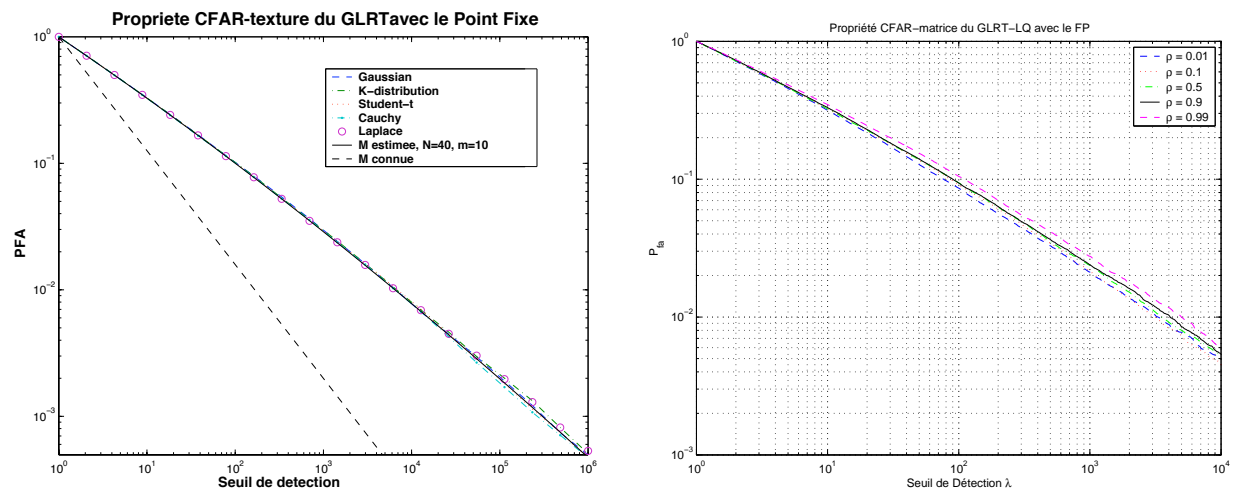

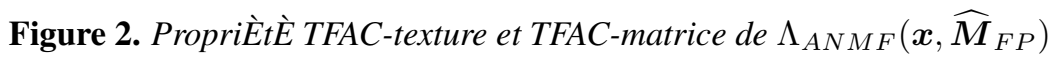

deuxiËme est la propriÈtÈ matrice-TFAC illustrÈe sur la mÍme figure ?? pour diffÈrentes matrice de covariance (ici matrices de Toeplitz du type $\boldsymbol{M}_{i, j}=\rho^{|i-j|}$ ). Enfin, la loi du test a pu Ítre Ètablie (Pascal et al., 2006) et donne explicitement la valeur du seuil de dÈtection $\lambda$ nÈcessaire $\ddagger$ la rÈgulation de fausse alarme fixÈe par la probabilitÈ de fausse alarme $P_{f a}$ :

$$
P_{f a}=(1-\lambda)^{a-1}{ }_{2} F_{1}(a, a-1 ; b-1 ; \lambda),
$$

avec $a=\frac{m}{m+1} K-m+2$ et $b=\frac{m}{m+1} K+2$.

\subsubsection{Applications sur donnÈes rÈelles et simulÈes}

\subsubsection{DonnÈes rÈelles}

Pour illustrer la puissance de ce dÈtecteur construit avec l'estimateur du point fixe, quelques rÈsultats issus de la rËgulation de la fausse alarme sur des donnÈes rÈelles provenant de THALES Air Defence sont prÈsentÈs. La gauche de la figure ?? reprËsente une carte "distance-azimut" d'Èchos de fouillis de sol collectÈs par un radar de TAD. Le radar, positionnÈ $\ddagger 13$ mËtres au-dessus du niveau de sol, Èclaire la zone avec un angle rasant. Les Èchos du sol ont ĖtÈ collectÈs pour $N=868$ cases distances, pour 70 angles d'azimut et pour $m=8$ impulsions. PrËs du radar, les Èchos caractËrisent un fouillis de sol hËtÈrogËne en puissance et non gaussien tandis qu'au del $\ddagger$ de l'horizon radio-Èlectrique du radar (environ 15 kilomËtres), seul le bruit thermique gaussien est prÈsent (partie de droite en bleue sur l'image).

Le seuil de dÈtection est rÈalisÈ par comptage, en dÈplaÁant un masque TFAC carrÈ de dimension $5 \times 5$. Pour chaque case centrale $\boldsymbol{x}$ du masque (i.e. la case testÈe), la valeur de $\Lambda_{A N M F}\left(\boldsymbol{x}, \widehat{\boldsymbol{M}}_{F P}\right)$ est calculÈe. La matrice de covariance $\widehat{\boldsymbol{M}}_{F P}$ est estimÈe sur les $K=24$ autres cases, considÈrÈs ainsi comme les donnÈes secondaires $\boldsymbol{c}_{k}$. Ce processus est rÈitÈrÈ pour chaque pixel sur la carte de fouillis, ici 

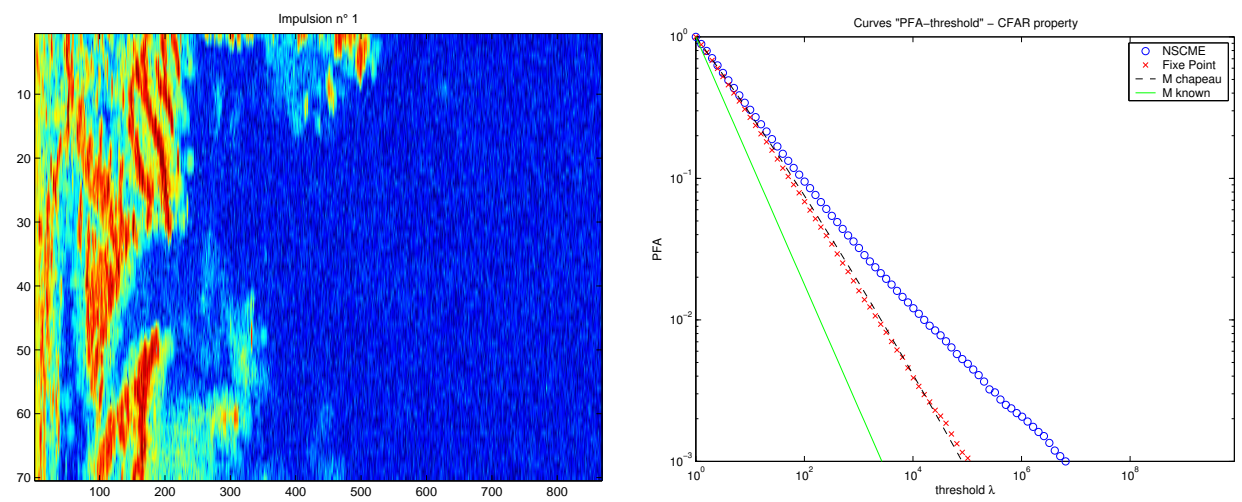

Figure 3. Figure de gauche : carte azimut-distance des Èchos radar de fouillis de sol. Figure de droite : comparaison des relations PFA-seuil pour l'ANMF construit avec diffÈrents estimateurs. a) Comptage Monte-Carlo de $\Lambda_{A N M F}\left(\boldsymbol{x}, \widehat{\boldsymbol{M}}_{N S C M}\right)$ en bleu b) Courbe thÈorique de l'Èquation [??] en noir. c) Courbe thÈorique correspondant $\ddagger$ l'idÈal (Èquation [??]) en vert. d) Comptage Monte-Carlo de $\Lambda_{A N M F}\left(\boldsymbol{x}, \widehat{\boldsymbol{M}}_{F P}\right)$ en rouge

$868 \times 70=60760$. Le comptage est ainsi effectuÈ et est prÈsentÈ $\ddagger$ la droite de la figure ??. Notons que la courbe de l'ANMF construit avec l'estimateur NSCM, $\Lambda_{A N M F}\left(\boldsymbol{x}, \widehat{\boldsymbol{M}}_{N S C M}\right)$ ne garantit pas la bonne rÈgulation de fausse alarme puisqu'il n'est pas matrice-TFAC. La courbe rouge du comptage des donnÈes effectuÈ avec l'ANMF construit avec le Point Fixe est en excellente adÈquation avec la thÈorie. La courbe verte est la courbe idÈale que l'on obtiendrait si la meilleure des estimations (cas $K \rightarrow \infty$ ) avait ÈtÈ rĖalisĚe (en fait, les performances pour $M$ connue). Cet Ècart avec l'estimation idÈale dÈfinit ce que l'on appelle en radar les pertes TFAC (CFAR loss). Ce dÈtecteur montre ici sa capacitÈ de robustesse vis $\ddagger$ vis de l'hÈtÈrogÈnÈitÈ de la zone sur laquelle sont collectÈes les donnÈes secondaires permettant d'estimer la matrice de covariance du fouillis.

\subsubsection{DonnÈes simulÈes}

Cette capacitÈ de robustesse aux transitions de fouillis est encore ici renforcÈe. Des simulations ont ĖtĖ rËalisÈes pour diffĖrentes zones de fouillis, allant du bruit gaussien $\ddagger$ un fouillis K-distribuÈ trËs impulsif. Pour chaque zone, le dËtecteur $\Lambda_{A N M F}\left(\boldsymbol{x}, \widehat{\boldsymbol{M}}_{F P}\right)$ est comparÈ $\ddagger \Lambda_{A M F}\left(\boldsymbol{x}, \widehat{\boldsymbol{M}}_{S C M}\right)$. La figure ?? de gauche prÈsente l'Ètape de rËgulation de fausses alarmes. La taille des vecteurs est $m=10$ et le nombre de donnÈes secondaires utilisĖes pour estimer la matrice de covariance est $K=20$. L'axe des ordonnËes correspond au seuil de dÈtection, l'axe des abscisses reprÈsente l'axe spatial (80 cases distance) correspondant $\ddagger$ diffĖrentes zones de fouillis : de $1 \ddagger 20$, le bruit est gaussien pour ensuite Ítre K-distribuÈ avec les paramËtres d'Èchelle $\nu$ suivants : de la case $20 \ddagger 40, \nu=2$, puis de $40 \ddagger 60, \nu=1$, de 
$60 \ddagger 80, \nu=0,5$, et enfin, de $80 \ddagger 100, \nu=0.1$ (pour des problËmes d'effet de bord, les cases $1 \ddagger 10$ et $90 \ddagger 100$ n'ont pas ÈtÈ reprËsentÈes). En fait, plus le paramËtre d'Èchelle diminue, plus le fouillis devient impulsif.
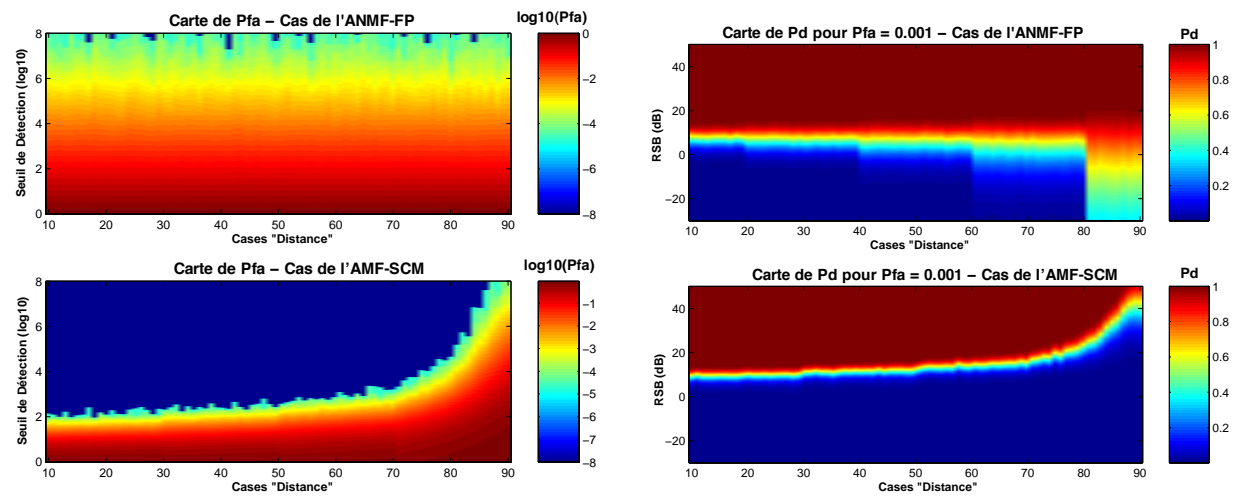

Figure 4. Analyse des performances de rÈgulation du taux de fausses alarmes et de dEtection pour l'AMF $\Lambda_{A M F}\left(\boldsymbol{x}, \boldsymbol{M}_{S C M}\right)$ et l'ANMF $\Lambda_{A N M F}\left(\boldsymbol{x}, \boldsymbol{M}_{F P}\right)$ dans 5 diffÈrentes zones de fouillis, allant du bruit gaussien $\ddagger$ un bruit trËs impulsif. a) $\ddagger$ gauche,

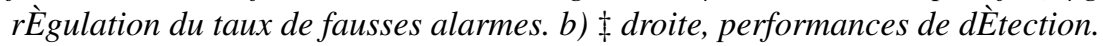

On peut immÈdiatement remarquer que le dÈtecteur $\Lambda_{A N M F}\left(\boldsymbol{x}, \widehat{\boldsymbol{M}}_{F P}\right)$, possÈdant la propriètÈ SIRV-TFAC, garantit la mÍme relation PFA-seuil dans toutes les zones de fouillis et est de ce fait robuste aux transitions. L'AMF a un comportement totalement diffÈrent : le seuil de dÈtection doit Ître rehaussÈ quand le bruit devient plus impulsif pour garantir le mÍme taux de fausses alarmes. La figure ?? de droite prÈsente les performances de dÈtection des deux dÈtecteurs. L'axe des ordonnÈes correspond au rapport signal-ł-bruit (RSB) tandis que l'axe des abscisses reprÈsente $\ddagger$ nouveau les 80 cases distances. La probabilitÈ de dÈtection $P_{d}$ pour une $P_{f a}$ rÈglÈe $\ddagger 10^{-3}$ est, elle aussi codÈe en couleur.

\section{Robustesse des Estimateurs}

Les nouveaux dÈtecteurs proposÈs plus haut dans le cas SIRV sont dits robustes car ils ne perdent pas leurs propriËtÈs TFAC lorsque l'on s'Ècarte lÈgËrement des hypothËses sous lesquelles ils ont ĖtÈ construits : robustesse $\ddagger l$ 'hÈtĖrogÈnÈitÈ spatiale et la non-gaussianitÈ du fouillis, robustesse aux "outliers" c'est $\ddagger$ dire $\ddagger$ la prËsence Èventuelles de cibles et de brouilleurs dans les cases adjacentes $\ddagger$ la case de test. De nouveaux travaux ont ainsi pu mettre en Èvidence l'intËrÍt d'emploi de la matrice FP du point fixe (mÍme en environnement gaussien) de par son trËs grande robustesse aux donnÈes aberrantes dans les algorithmes de type Music ou Esprit ou dans la mise en œuvre des dÈtecteurs radar (?). 
Les donnÈes secondaires sont supposÈes toujours respecter le modËle, c'est $\ddagger$ dire que les donnÈes secondaires dans le cas de la dÈtection contiennent toujours un bruit respectant les hypothËses de dËpart. On considËre ici le problËme d'estimation de la matrice de covariance du processus Ėtant donnÈ $N$ observations secondaires indÈpendantes $\left\{\boldsymbol{x}_{k}\right\}_{k=1 . . N}$ contenant du bruit et quelques perturbations additives. Quand $K$ observations $\left\{\boldsymbol{x}_{k}\right\}_{k=1 . . K}$ contiennent de fortes perturbations dÈterministes $\left\{\boldsymbol{a}_{k}\right\}_{k=1 . . K}$ et un bruit additif $\left\{\boldsymbol{c}_{k}\right\}_{k=1 . . K}$, on peut analyser le biais (?) entre l'estimation de la matrice de covariance contaminËe et l'estimation de la matrice non contaminÈe. Supposons ici, pour des raisons de comprÈhension, que $\left\|\boldsymbol{a}_{k}\right\|>>\left\|\boldsymbol{c}_{k}\right\|$ et que les $\left\{\boldsymbol{c}_{k}\right\}$ soient gaussiens.
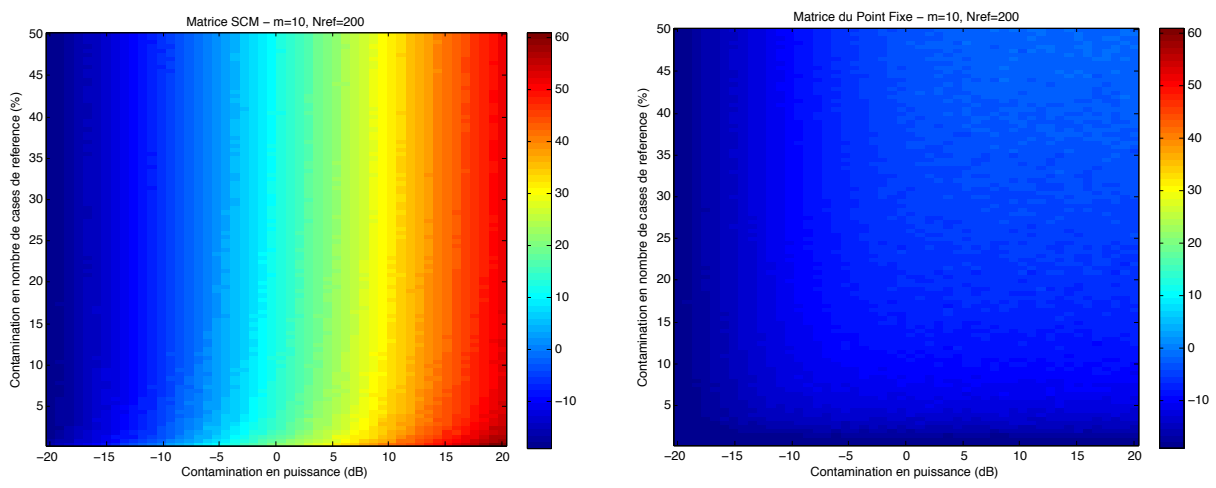

Figure 5. Erreur relative (en $d B$ ) entre la matrice de covariance estimÈe non contaminÈe et la matrice de covariance estiṁ̀e contaminÈe. a) $\ddagger$ gauche, matrice SCM conventionnelle. $b) \ddagger$ droite, matrice du point fixe.

\subsection{Analyse du biais de la SCM en prÈsence de perturbations :}

La diffÈrence $\Delta_{S C M}$ entre l'espÈrance statistique de la SCM contaminÈe et l'espÈrance statistique $E\left[\widehat{M}_{\mathrm{SCM}}\right]=M$ de la SCM non contaminÈe, soit le biais $\mathrm{d}^{\circ} \ddagger$ la contamination, s'Ècrit :

$$
\Delta_{S C M}=\frac{m}{N} \sum_{k=1}^{K} \boldsymbol{a}_{k} \boldsymbol{a}_{k}^{H}-\frac{K}{N} \boldsymbol{M} .
$$

On peut noter que plus la norme $\left\|\boldsymbol{a}_{k}\right\|$ des perturbations est forte, plus le biais est important. La matrice estimÈe $\widehat{\boldsymbol{M}}_{\mathrm{SCM}}$ n'est donc pas robuste. 

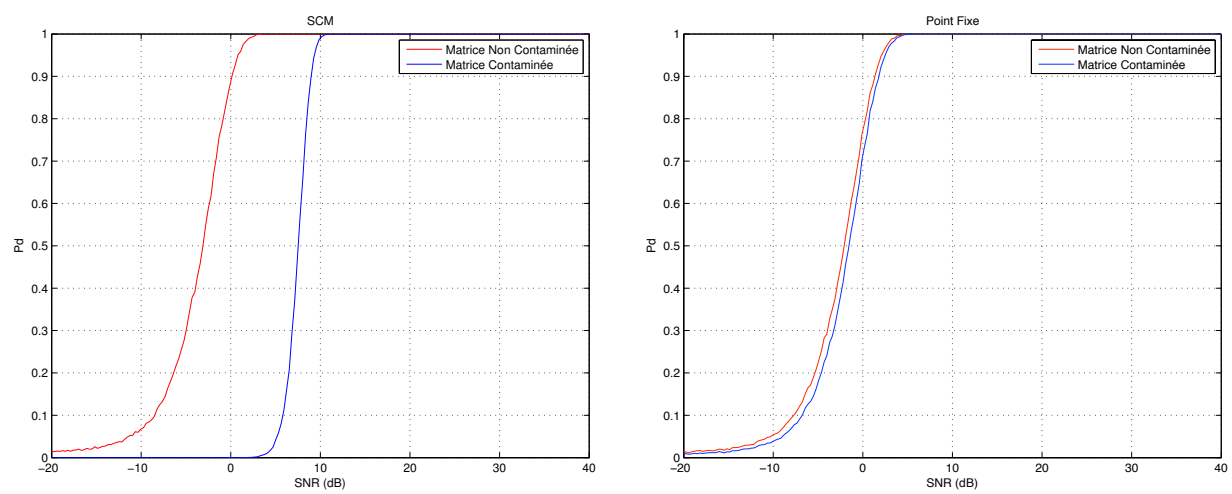

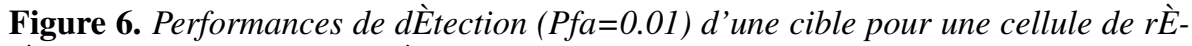
fËrence fortement contaminÈe $(20 \mathrm{~dB})$ ou pas. a) $\ddagger$ gauche, performances de l'AMF avec SCM biaisÈe ou pas par la contamination. $b$ ) $\ddagger$ droite, performances de l'ANMF avec l'estimateur robuste du point fixe

\subsection{Analyse du biais de la NSCM en prèsence de perturbations :}

La diffÈrence $\Delta_{N S C M}$ entre l'espÈrance statistique de la NSCM contaminÈe et l'espÈrance statistique $E\left[\widehat{\boldsymbol{M}}_{\mathrm{NSCM}}\right]$ de la NSCM non contaminÈe ( $\neq M$ car l'estimateur NSCM est biaisÈ), soit le biais d $\mathrm{d}^{\circ} \ddagger$ la contamination, s'Ècrit :

$$
\Delta_{N S C M}=-\frac{K}{N} E\left[\widehat{\boldsymbol{M}}_{\mathrm{NSCM}}\right]+\frac{m}{N} \sum_{k=1}^{K} \frac{\boldsymbol{a}_{k} \boldsymbol{a}_{k}^{H}}{\boldsymbol{a}_{k}^{H} \boldsymbol{a}_{k}} .
$$

\subsubsection{Analyse du biais de la FP en prèsence de perturbations :}

Dans ce cas, le biais $\Delta_{F P}=E\left[\widehat{\boldsymbol{M}}_{\mathrm{FP}}\right]-\boldsymbol{M}$ de la matrice du point fixe contaminÈe s'Ècrit

$$
\Delta_{F P}=\frac{m+1}{N}\left(\sum_{k=1}^{K} \frac{\boldsymbol{a}_{k} \boldsymbol{a}_{k}^{H}}{\boldsymbol{a}_{k}^{H} \boldsymbol{M}^{-1} \boldsymbol{a}_{k}}-\frac{K}{m} \boldsymbol{M}\right) .
$$

Dans les deux derniers cas, on peut remarquer que la force $\left\|\boldsymbol{a}_{k}\right\|$ de la contamination n'influe pas dans le calcul de ces biais. Tout facteur d'Ëchelle scalaire sur les vecteurs $\left\{\boldsymbol{a}_{k}\right\}$ laisse le bais invariant. Les estimateurs NSCM et FP sont donc robustes $\ddagger$ la prËsence de perturbations dÈterministes.

La figure ?? montre l'erreur relative que l'on commet pour l'estimateur classique SCM et l'estimateur robuste du point fixe lorsqu' on estime la covariance avec des donnÈes de rÈfÈrence contaminÈes (en nombre et en puissance). La figure ?? donne une comparaison des performances entre une technique conventionnelle et une technique 
robuste pour une seule cellule contaminÈe dans les donnÈes de rÈfÈrence contenant une cible trËs forte de $20 \mathrm{~dB}$ (cible de mÍme steering vector que dans la case de test, cas, par exemple, d'un convoi ÈtalÈ spatialement de plusieurs vÈhicules de mÍmes vitesses). L'axe des abscisses dÈfinit la puissance des contaminations et l'axe des ordonnÈes dÈfinit le nombre de cellules contaminÈes (sur 200 cases).

Cette robustesse est Ègalement mis en Èvidence dans (?)†dans le cadre du STAP $\ddagger$ rang rÈduit lorsque les donnÈes secondaires contiennent des cibles de mÍme steering vector (cas de convois par exemple). Lors du processus de blanchiment, la matrice de covariance SCM estimÈe avec de fortes cibles prÈsentes dans les donnÈes secondaires blanchit Ègalement la cible dans la case de test. L'utilisation de la NSCM ou du FP permet d'attËnuer trËs fortement l'influence de leur prËsence et confËre ainsi au dÈtecteur associè une grande robustesse.

Cette problÈmatique, peu connue dans le monde radar, est ÈtudiÈe depuis de nombreuses annÈes en statistique sous le nom de Statistiques Robustes (?; ?; ?). On y rencontre notamment les $M$-estimateurs, estimateurs robustes de matrice de covariance dans le cadre beaucoup plus Ėtendu des processus elliptiques (?), qui contiennent naturellement les processus SIRV. Les $M$-estimateurs sont dÈfinis comme la solution de l'Èquation implicite :

$$
\widehat{\boldsymbol{M}}=\frac{1}{K} \sum_{i=1}^{K} u\left(\boldsymbol{c}_{i}^{H} \widehat{\boldsymbol{M}}^{-1} \boldsymbol{c}_{i}\right) \boldsymbol{c}_{i} \boldsymbol{c}_{i}^{H},
$$

o $u($.$) est une fonction devant respecter quelques contraintes. On peut montrer que ces$ estimateurs (ou des fonctionnelles continues de ces estimateurs), $\ddagger$ une normalisation adÈquate prËs, ont les mÍmes performances asymptotiques que celle de la matrice SCM, ceci pour un nombre de donnÈes secondaires lÈgËrement supÈrieur (?; ?).

Les recherches que nous menons actuellement portent sur la prise en compte de la non-stationnaritÈ de case distance $\ddagger$ case distance de cette matrice de covariance que l'on rencontre en STAP de pointe avant par exemple ou sur du fouillis dynamique comme la mer.

\section{Utilisation de la structure de Covariance en dÈtection STAP}

Les techniques de dÈtection prÈsentÈes prÈcÈdemment ont ÈtÈ Ètendues (Pailloux et al., 2008; 2009; 2010; 2011) pour rÈpondre au problËme crucial de la rÈduction du nombre de cases distance (rËduction du nombre $K$ de donnÈes secondaires) nÈcessaires $\ddagger$ l'estimation de la matrice de covariance du fouillis. Nous nous sommes ainsi appuyÈs sur l'information a priori que l'on pouvait apporter $\ddagger$ la matrice de covariance. La prise en compte de la structure particuliËre de Toeplitz de la matrice de covariance a ĖtÈ largement ĖtudiÈe par (?) dans le cadre de l'estimation et par (?) dans le cadre de la dËtection radar. En utilisant la propriÈtĖ dite de persymËtrie (symÈtrie par rapport $\ddagger 1$ 'antidiagonale) de cette matrice que l'on rencontre en STAP latĖral conventionnel, dans tout type de radar $\ddagger$ pÈriode de rÈpÈtition constante 
ou dans toute application d'antenne de type ULA (Uniform Linear Antenna), (?) et (?) ont respectivement proposÈ un estimateur du Maximum de Vraisemblance de la matrice de covariance et son dÈtecteur GLRT associÈ. Dans le cadre des SIRV, des schÈmas de dÈtection ont ĖtĖ proposÈs par (?) et (?). Dans (?), la persymĖtrie est seulement exploitËe pour construire deux jeux indĖpendants de donnËes secondaires nÈcessaires $\ddagger$ l'Èlaboration d'un dÈtecteur SIRV-TFAC, le Persymmetric Adaptive Normalized Matched Filter (P-ANMF). Dans (?), les jeux de donnÈes prÈcÈdents sont utilisÈs pour initialiser l'algorithme itĖratif proposÈ dans (?; ?) et pour construire le dÈtecteur rÈcursif RP-ANMF (Recursive P-ANMF).

Notre approche exploite une transformation originale dÈjł prÈsentÈe dans (?) pour le cas gaussien et dans (?). Pour le cas gaussien, il est possible de dÈfinir un dÈtecteur de type AMF construit sur l'estimateur du Maximum de Vraisemblance de la matrice de covariance persymÈtrique : le PS-AMF (Persymmetric Adaptive Matched Filter). Pour le cas SIRV, la mÈthodologie est basÈe sur l'utilisation conjointe de la matrice du point fixe (FP) et de l'ANMF, pour dĖfinir le Fixed Point Adaptive Normalized Matched with Persymmetric Fixed Point (ANMF-PFP ou GLRT-PFP).

L'intÈrÍt de ces deux approches rÈside dans le fait que, dans les deux cas, la matrice estimÈe est rÈelle plutÙt que complexe, conduisant $\ddagger$ un nombre de degrÈs de libertÈ deux fois moins important. À nombre $K$ de donnÈes secondaires donnÈ, ces deux dÈtecteurs, couplÈs $\ddagger$ l'estimateur associÈ amÈliore fortement les performances de dÈtection.

Soit $\mathbf{M}$ une matrice de covariance. Cette matrice $\mathbf{M}$ possËde la propriÈtÈ de persymÈtrie si et seulement si :

$$
\boldsymbol{M}=\boldsymbol{J}_{m} \boldsymbol{M}^{*} \boldsymbol{J}_{m},
$$

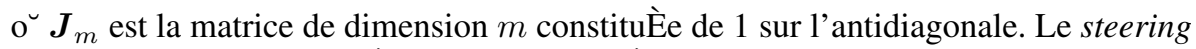
vector $\boldsymbol{a}$ du problËme est Ègalement persymĖtrique si et seulement si :

$$
\boldsymbol{a}=\boldsymbol{J}_{m} \boldsymbol{a}^{*} .
$$

Cette propriÈtÈ de persymÈtrie caractÈrisant la matrice de covariance des signaux, peut s'exploiter en considÈrant la transformation des donnÈes par une matrice unitaire $\boldsymbol{T}$ donnÈe par :

$$
\boldsymbol{T}= \begin{cases}\frac{1}{\sqrt{2}}\left(\begin{array}{cc}
\boldsymbol{I}_{m / 2} & \boldsymbol{J}_{m / 2} \\
i \boldsymbol{I}_{m / 2} & -i \boldsymbol{J}_{m / 2}
\end{array}\right) & \text { pour } m \text { pair } \\
\frac{1}{\sqrt{2}}\left(\begin{array}{ccc}
\boldsymbol{I}_{(m-1) / 2} & 0 & \boldsymbol{J}_{(m-1) / 2} \\
0 & \sqrt{2} & 0 \\
i \boldsymbol{I}_{(m-1) / 2} & 0 & -i \boldsymbol{J}_{(m-1) / 2}
\end{array}\right) & \text { pour } m \text { impair }\end{cases}
$$

o $\boldsymbol{I}_{m}$ est la matrice unitÈ de dimension $m$. Les vecteurs et matrices persymÈtriques sont caractËrisÈs par les propriÈtÈs suivantes :

- $\boldsymbol{a} \in \mathbb{C}^{m}$ est un vecteur persymÈtrique si et seulement si $\boldsymbol{T} \boldsymbol{a}$ est un vecteur rÈel. 
- $\boldsymbol{M}$ est une matrice hermitienne persymÈtrique si et seulement si $\boldsymbol{T} \boldsymbol{M} \boldsymbol{T}^{H}$ est une matrice symÈtrique rÈelle.

Notons $\boldsymbol{y}, \boldsymbol{y}_{k}, \boldsymbol{n}$ et $\boldsymbol{s}$ les donnÈes du problËme [??] aprËs la transformation $\boldsymbol{T}$ : $\boldsymbol{y}=\boldsymbol{T} \boldsymbol{x}, \boldsymbol{y}_{k}=\boldsymbol{T} \boldsymbol{x}_{k}, \boldsymbol{s}=\boldsymbol{T} \boldsymbol{a}, \boldsymbol{n}=\boldsymbol{T} \boldsymbol{c}, \boldsymbol{n}_{k}=\boldsymbol{T} \boldsymbol{c}_{k}$. Il s'ensuit que le steering vector $s$ et la matrice $\boldsymbol{T}$ sont tous deux rËels. Le problËme original de dËtection prÈsentÈ en [??] peut alors se transformer de maniËre Èquivalente :

$$
\begin{cases}H_{0}: \boldsymbol{y}=\boldsymbol{n} & \boldsymbol{y}_{k}=\boldsymbol{n}_{k}, \text { for } 1 \leq k \leq K \\ H_{1}: \boldsymbol{y}=\alpha \boldsymbol{s}+\boldsymbol{n} & \boldsymbol{y}_{k}=\boldsymbol{n}_{k}, \text { for } 1 \leq k \leq K\end{cases}
$$

o $\boldsymbol{y} \in \mathbb{C}^{m}$ et $\boldsymbol{s}$ est un vecteur rèel connu.

Dans le cas gaussien et sous l'hypothËse $H_{0}, \boldsymbol{y}$ et les $K$ donnÈes secondaires transformÈes $\boldsymbol{y}_{k}$ sont identiquement distribuÈes et partagent la mÍme distribution

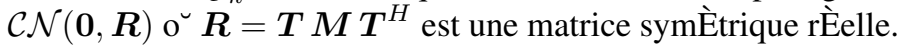

Dans le cas SIRV, on a :

$$
\boldsymbol{n}=\sqrt{\tau} \boldsymbol{h}, \quad \boldsymbol{n}_{k}=\sqrt{\tau_{k}} \boldsymbol{h}_{k},
$$

o $\boldsymbol{h}=\boldsymbol{T} \boldsymbol{g}$ et $\boldsymbol{h}_{k}=\boldsymbol{T} \boldsymbol{g}_{k}$ sont les vecteurs de speckle transformÈs et caractÈrisÈs par la mÍme matrice de covariance rÈelle $\boldsymbol{R}=\boldsymbol{T} \boldsymbol{M} \boldsymbol{T}^{H}$. Gr,ce $\ddagger$ la propriÈtÈ d'invariance des SIRV par filtrage linÈaire (?), les vecteurs $\boldsymbol{n}$ et $\boldsymbol{n}_{k}$ restent encore SIRV aprËs la transformation avec la mÍme loi de texture $p_{\tau}($.$) mais avec la nouvelle ma-$ trice de covariance $\boldsymbol{R}=\boldsymbol{T} \boldsymbol{M} \boldsymbol{T}^{H}$. Le nouveau problëme $\ddagger$ rÈsoudre est ainsi celui dÈfini en (??).

\subsection{Cas gaussien}

Le problËme est ici de dÈterminer, dans le cas gaussien, l'estimÈe du Maximum de Vraisemblance de la matrice de covariance $\boldsymbol{R} \ddagger$ l'aide des $K$ donnÈes secondaires $\boldsymbol{y}_{k}$.

L'estimÈe $\widehat{\mathbf{R}}_{P}$ au sens du Maximum de Vraisemblance de la matrice de covariance $\boldsymbol{R}$ est donnÈe par :

$$
\widehat{\mathbf{R}}_{P}=\mathcal{R} e\left(\widehat{\mathbf{R}}_{S C M}\right),
$$

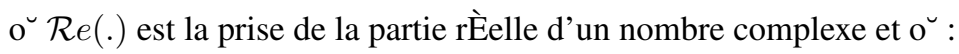

$$
\widehat{\mathbf{R}}_{S C M}=\frac{1}{K} \sum_{k=1}^{K} \mathbf{x}_{k} \mathbf{x}_{k}^{H}=\mathbf{T} \widehat{\mathbf{M}}_{S C M} \mathbf{T}^{H} .
$$

$\widehat{\mathbf{R}}_{P}$ est non biaisÈ et $K \widehat{\mathbf{R}}_{P}$ est rÈel, Wishart distribuÈ de paramËtre $\frac{1}{2} \mathbf{R}$ et $2 K$ degrÈs de libertÈ. 
Ainsi, le fait de prendre en compte la structure rÈelle de $\boldsymbol{R}$ (ou de maniËre Èquivalente la structure persymÈtrique de $\boldsymbol{M}$ ) dans le processus d'estimation, permet de virtuellement doubler le nombre $K$ de donnÈes secondaires, ce qui n'est pas nÈgligeable ‡ faible nombre de donnÈes secondaires (assouplissement de la rËgle de Brennan).

Le dÈtecteur [??] de type AMF sur le problËme dÈfini par [??] et basè sur l'estimateur $\widehat{\boldsymbol{R}}_{P}$ dÈfini par [??] est appelÈ PS-AMF (Persymmetric AMF) et prend la forme :

$$
\Lambda_{P S-A M F}\left(\widehat{\boldsymbol{R}}_{P}, \boldsymbol{y}\right)=\frac{\left|\boldsymbol{s}^{\top} \widehat{\boldsymbol{R}}_{P}^{-1} \boldsymbol{y}\right|^{2}}{\boldsymbol{s}^{\top} \widehat{\boldsymbol{R}}_{P}^{-1} \boldsymbol{s}} \underset{H_{0}}{\stackrel{H_{1}}{\gtrless}} \lambda_{P S-A M F},
$$

ou de maniËre Èquivalente en fonction des donnÈes initiales :

$$
\Lambda_{P S-A M F}\left(\widehat{\boldsymbol{M}}_{S C M}, \boldsymbol{x}\right)=\frac{\left|\boldsymbol{a}^{H} \boldsymbol{T}^{H}\left[\mathcal{R} e\left(\boldsymbol{T} \widehat{\boldsymbol{M}}_{S C M} \boldsymbol{T}^{H}\right)\right]^{-1} \boldsymbol{T} \boldsymbol{x}\right|^{2}}{\boldsymbol{a}^{H} \boldsymbol{T}^{H}\left[\mathcal{R} e\left(\boldsymbol{T} \widehat{\boldsymbol{M}}_{S C M} \boldsymbol{T}^{H}\right)\right]^{-1} \boldsymbol{T} \boldsymbol{a}}{\underset{H_{0}}{\gtrless}}_{H_{P S-A M F}} .
$$

La distribution du test [??] est connue lorsque $K \widehat{\boldsymbol{R}}_{P}$ est complexe, Wishart distribuÈ $\mathcal{C W}_{m}(K \boldsymbol{R}, K)$ et correspond $\ddagger$ celle classique de l'AMF (?) donnÈe par [??] . Dans notre problËme, $K \widehat{\boldsymbol{R}}_{P}$ est rÈel, Wishart distribuÈ de paramËtre $\frac{1}{2} \boldsymbol{R}$ et $2 K$ degrÈs de libertÈ tandis que $\boldsymbol{y}$ est complexe. Il est possible d'Ètablir la distribution du PS-AMF et la relation analytique liant la PFA $P_{f a}$ et le seuil de dÈtection $\lambda_{P S-A M F}$.

- Sous hypothËse $H_{0}$, la distribution $p(z)$ de $\Lambda_{P S-A M F}$ dÈfini par [??], prend la forme :

$$
\begin{gathered}
p(z)=\frac{(2 K-m+1)(2 K-m+2)}{2 K(2 K+1)} \\
{ }_{2} F_{1}\left(\frac{2 K-m+3}{2}, \frac{2 K-m+4}{2} ; \frac{2 K+3}{2} ;-\frac{z}{K}\right),
\end{gathered}
$$

- La relation liant la $P_{f a}$ et le seuil de dÈtection $\lambda$ est donnÈe par :

$$
P_{f a}={ }_{2} F_{1}\left(\frac{2 K-m+1}{2}, \frac{2 K-m+2}{2} ; \frac{2 K+1}{2} ;-\frac{\lambda_{P S-A M F}}{K}\right) .
$$

Comme nous le verrons dans la partie expÈrimentation, le PS-AMF donne de bien meilleures performances que l'AMF, notamment dans le cas d'un petit nombre $K$ de donnÈes secondaires.

\subsection{Cas SIRV}

Le problËme est ici de dÈterminer, dans le cas SIRV, l'estimÈe du Maximum de Vraisemblance de la matrice de covariance $\boldsymbol{R} \ddagger$ l'aide des $K$ donnÈes secondaires 
$\boldsymbol{y}_{k}$ et d'Ètablir le dÈtecteur associÈ au test d'hypothËses (??). Nous rappelons que le bruit additif SIRV $\boldsymbol{n}$ est donnÈ par :

$$
\boldsymbol{n}=\sqrt{\tau} \boldsymbol{h},
$$

$\mathrm{o}^{\tau}$ est une variable alÈatoire scalaire positive, et $\boldsymbol{h}$ est un vecteur centrÈ gaussien de matrice de covariance $\boldsymbol{R}$. TLes $K$ donnÈes secondaires $\boldsymbol{n}_{k}=\sqrt{\tau_{k}} \boldsymbol{h}_{k}$ sont identiquement distribuÈ et partage la mÍme distribution que $\boldsymbol{n}$.

Puisque la matrice transformÈe $\boldsymbol{R}$ est rÈelle, sa structure doit Ítre prise en considÈration dans la procÈdure d'estimation en ne retenant que la partie rÈelle de l'estimateur du point fixe. Ceci conduit $\ddagger$ proposer un nouvel estimateur de covariance appelÈ Persymmetric Fixed Point (PFP) et dÈfini par :

$$
\widehat{\boldsymbol{R}}_{P F P}=\mathcal{R} e\left(\widehat{\boldsymbol{R}}_{F P}\right),
$$

avec

$$
\widehat{\boldsymbol{R}}_{F P}=\boldsymbol{T} \widehat{\boldsymbol{M}}_{F P} \boldsymbol{T}^{H} .
$$

Les propriÈtÈs intrinsËques et statistiques de $\widehat{\boldsymbol{R}}_{P F P}$ peuvent Ítre donnÈes :

- La distribution de $\widehat{\boldsymbol{R}}_{P F P}$ ne dÈpend pas de la texture.

- $\widehat{\boldsymbol{R}}_{P F P}$ est un estimateur consistant de $\boldsymbol{R}$.

- $\widehat{\boldsymbol{R}}_{P F P}$ est un estimateur non-biaisÈ de $\boldsymbol{R}$.

- $\frac{\widehat{\boldsymbol{R}}_{P F P}}{\operatorname{tr}\left\{\boldsymbol{R}^{-1} \widehat{\boldsymbol{R}}_{P F P}\right\}}$ possëde la mÍme distribution que $\frac{\widehat{\boldsymbol{R}}}{\operatorname{tr}\left\{\boldsymbol{R}^{-1} \widehat{\boldsymbol{R}}\right\}}$, ○ $\widehat{\boldsymbol{R}}$ est rÈel, Wishart distribuÈ $\ddagger \frac{m}{m+1} 2 K$ degrÈs de libertÈ et de paramËtre $\boldsymbol{R}$.

Un nouveau dÈtecteur associÈ au problËme [??], appelÈ ANMF-PFP Adaptive Normalized Matched Filter - Persymmetric Fixed Point et basÈ sur [??] et sur l'estimateur PFP [??], prend la forme :

$$
\Lambda_{A N M F-P F P}\left(\widehat{\boldsymbol{R}}_{P F P}, \boldsymbol{y}\right)=\frac{\left|\boldsymbol{s}^{\top} \widehat{\boldsymbol{R}}_{P F P}^{-1} \boldsymbol{y}\right|^{2}}{\left(\boldsymbol{s}^{\top} \widehat{\boldsymbol{R}}_{P F P}^{-1} \boldsymbol{s}\right)\left(\boldsymbol{y}^{H} \widehat{\boldsymbol{R}}_{P F P}^{-1} \boldsymbol{y}\right)}{\underset{H}{H_{0}}}_{H_{1}}^{\gtrless} \lambda_{A N M F-P F P},
$$

ou de maniËre Èquivalente avec les donnÈes initiales :

$$
\begin{gathered}
\Lambda_{A N M F-P F P}\left(\widehat{\boldsymbol{M}}_{F P}, \boldsymbol{x}\right)= \\
\frac{\left|\boldsymbol{a}^{H} \boldsymbol{T}^{H}\left[\mathcal{R e}\left(\boldsymbol{T} \widehat{\boldsymbol{M}}_{F P} \boldsymbol{T}^{H}\right)\right]^{-1} \boldsymbol{T} \boldsymbol{x}\right|^{2}}{\left(\boldsymbol{x}^{H} \boldsymbol{T}^{H}\left[\mathcal{R} e\left(\boldsymbol{T} \widehat{\boldsymbol{M}}_{F P} \boldsymbol{T}^{H}\right)\right]^{-1} \boldsymbol{T} \boldsymbol{x}\right)\left(\boldsymbol{a}^{H} \boldsymbol{T}^{H}\left[\mathcal{R} e\left(\boldsymbol{T} \widehat{\boldsymbol{M}}_{F P} \boldsymbol{T}^{H}\right)\right]^{-1} \boldsymbol{T} \boldsymbol{a}\right)} .
\end{gathered}
$$

Ce dÈtecteur possËde quelques propriÈtÈs trËs intÈressantes : 
$-\Lambda_{A N M F-P F P}$ est SIRV-TFAC,

- Pour $K$ assez grand, sous hypothËse $H_{0}, \Lambda_{A N M F-P F P}$ possËde la mÍme

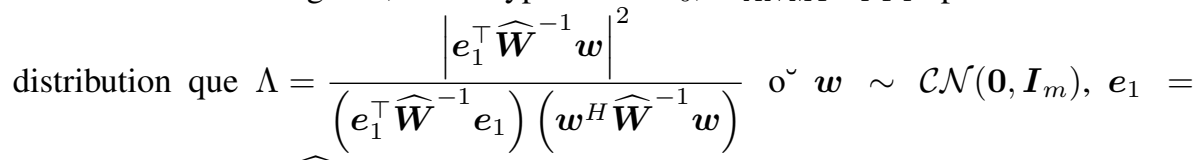
$(1,0, \ldots, 0)^{\top}$ et o $\widehat{\boldsymbol{W}}$ est rÈelle, Wishart distribuè de paramËtre $\boldsymbol{I}_{m}$ et $K^{\prime}=$ $\frac{m}{m+1} 2 K$ degrÈs de libertÈ. Cette distribution n'est pas connue analytiquement mais on peut donner quelques indications et identifier les variables indÈpendantes la constituant : pour $K$ assez grand, $\Lambda_{A N M F-P F P}$ possËde la mÍme distribution que $\frac{F}{F+1}$ o :

$$
F=\frac{\left(\alpha_{1} l_{22}-\alpha_{2} l_{21}\right)^{2}+\left(1+\left(\frac{\beta_{3}}{l_{33}}\right)^{2}\right)\left(a l_{22}-b l_{21}\right)^{2}}{\left(\alpha_{2} l_{11}\right)^{2}+\left(l_{11} l_{22} \frac{\beta_{3}}{l_{33}}\right)^{2}+l_{11}^{2}\left(1+\left(\frac{\beta_{3}}{l_{33}}\right)^{2}\right) b^{2}},
$$

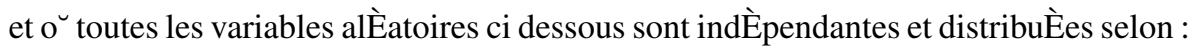

$$
a, b, \alpha_{1}, l_{21} \sim \mathcal{N}(0,1), \alpha_{2}^{2} \sim \chi_{m-1}^{2}, \beta_{3}^{2} \sim \chi_{m-2}^{2},
$$

et

$$
l_{11}^{2} \sim \chi_{K^{\prime}-m+1}^{2}, l_{22}^{2} \sim \chi_{K^{\prime}-m+2}^{2}, l_{33}^{2} \sim \chi_{K^{\prime}-m+3}^{2},
$$

with $K^{\prime}=\frac{m}{m+1} 2 K$.

Cette derniËre propriÈtÈ permet, $\ddagger$ travers des tirages de type Monte Carlo, d’Èlaborer trËs facilement les cartes PFA-seuil de dÈtection de $\lambda_{A N M F-P F P}$ [??].

À nombre de vecteurs secondaires donnÈ, ces techniques amÈliorent encore les performances des dÈtecteurs conventionnels de type AMF + SCM (nouveau dÈtecteur PS-AMF) ou les dËtecteurs de type ANMF+FP (nouveau dÈtecteur ANMF-PFP) (?). Cette technique de persymĖtrie aliÈe ou non aux processus SIRV peut Ègalement Ítre mise en œuvre de maniËre trËs performante dans les dËtecteurs STAP $\ddagger$ rang rÈduit comme le montre (?).

\subsection{Applications sur donnèes rèelles}

La figure ?? donne un exemple de rÈsultat obtenu sur les donnÈes de THALES de la figure ?? pour $m=8$ et $K=8$, soit un nombre $K$ de donnÈes secondaires Ègal $\ddagger$ la dimension $m$ de la matrice de covariance. En bleu foncÈ, sont prËsentÈes la courbe de fausses alarmes pour une covariance thÈoriquement connue, en rose et rouge, les courbes de fausses alarmes thÈorique et estimÈe (simulation) du dÈtecteur ANMF n'utilisant pas la propriÈtÈ de persymÈtrie de la matrice, en noir et bleu clair, 
les performances apportÈes par l'information a priori de la persymÈtrie dans le dÈtecteur ANMF-PFP. La figure ?? donne le mÍme genre d'exemple pour $m=8$ et $K=20$, soit un nombre $K$ de donnÈes secondaires supÈrieur au double de la dimension $m$ de la matrice de covariance. Sur chaque figure, sont reprÈsentÈs les gains de performance de dÈtection. Il est clair qu' $\ddagger$ un nombre de donnËes secondaires $K$ Ègal $\ddagger$ la dimension $m$ du vecteur de donnÈes (voir figure ??), les performances sont grandement amÈliorÈes.
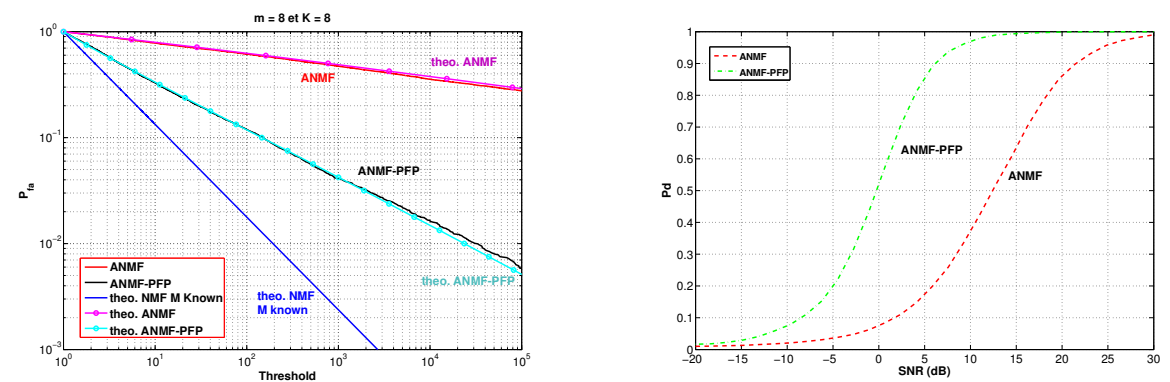

Figure 7. Courbes de rÈgulation de fausse alarme et de performance de ḋ̀tection sur les donnÈes de THALES (seuil de dÈtection $\eta=(1-\lambda)^{-m}$ en fonction de la probabilitè de fausse alarme) pour les dÈtecteurs $\Lambda_{A N M F}\left(\widehat{\boldsymbol{M}}_{F P}, \boldsymbol{x}\right)$ et $\Lambda_{A N M F-P F P}\left(\widehat{\boldsymbol{M}}_{F P}, \boldsymbol{x}\right)$. Cas $P_{f a}=10^{-2}, m=8$ et $K=8$
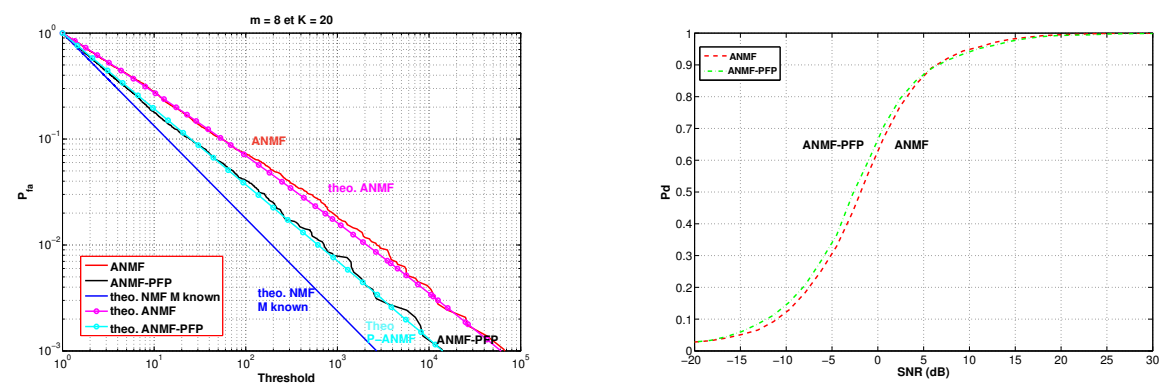

Figure 8. Courbes de rÈgulation de fausse alarme et de performance de dÈtection sur les donnÈes de THALES (seuil de dÈtection $\eta=(1-\lambda)^{-m}$ en fonction de la probabilitל̀ de fausse alarme) pour les dÈtecteurs $\Lambda_{A N M F}\left(\widehat{\boldsymbol{M}}_{F P}, \boldsymbol{x}\right)$ et $\Lambda_{A N M F-P F P}\left(\widehat{\boldsymbol{M}}_{F P}, \boldsymbol{x}\right)$. Cas $P_{f a}=10^{-2}, m=8$ et $K=20$

\section{Applications sur donnÈes STAP}

La figure ?? montre les rÈsultats obtenus sur des donnÈes STAP synthÈtisÈes par la DGA MaÓtrise de l'information $\ddagger$ partir d'images rËelles SAR du capteur SETHI 


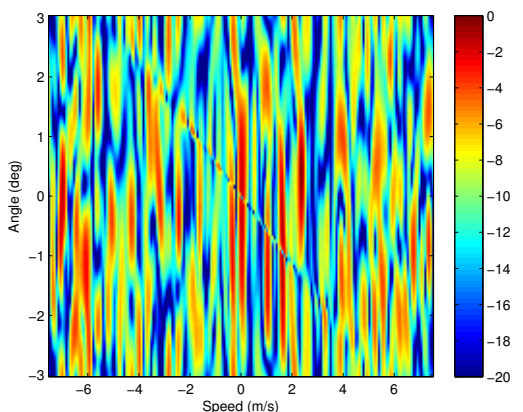

(a) $\Lambda_{A M F}\left(\widehat{\boldsymbol{M}}_{S C M}, \boldsymbol{x}\right)$

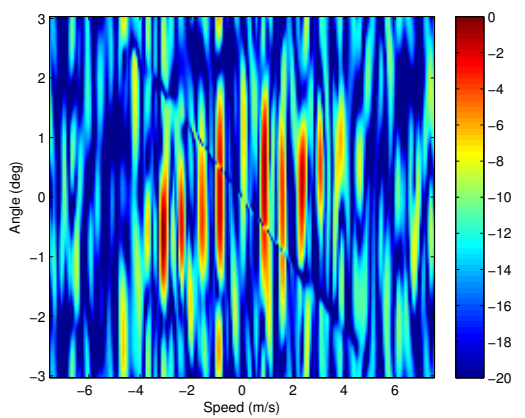

(c) $\Lambda_{A N M F}\left(\widehat{\boldsymbol{M}}_{F P}, \boldsymbol{x}\right)$

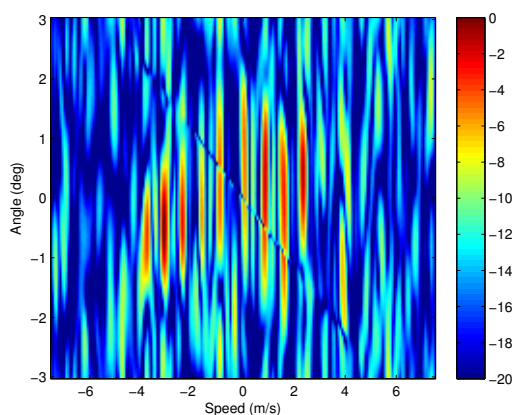

(b) $\Lambda_{P S-A M F}\left(\widehat{\boldsymbol{M}}_{S C M}, \boldsymbol{x}\right)$

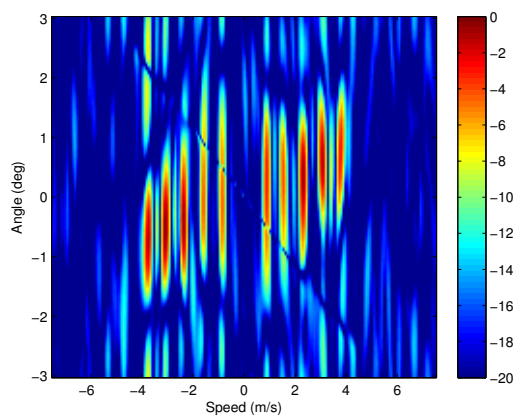

(d) $\Lambda_{A N M F-P F P}\left(\widehat{\boldsymbol{M}}_{F P}, \boldsymbol{x}\right)$

Figure 9. RÈsultats de dÈtection Doppler-azimut dans la case distance 255 pour 10 cibles de vitesses diffÈrentes ( $N M=512, K=410$.

de l'ONERA. Dix cibles artificielles dans la mÍme case distance (case 255) mais $\ddagger$ des vitesses diffÈrentes ont ainsi ÈtÈ ajoutÈes dans les donnÈes synthÈtisÈes. La dimension des vecteurs STAP est ici de $m=M N=256$ alors que le nombre de donnÈes secondaires est $K=410$. La rËgle de Brennan $(m>2 K$ ) et qui garantit, dans le cas gaussien, une perte maximale de $3 \mathrm{~dB}$, n'est pas respectÈe. L'utilisation de la propriËtÈ de persymËtrie permet ainsi d'utiliser virtuellement $2 \mathrm{~K}$ donnËes secondaires et on peut remarquer que les dÈtecteurs persymÈtriques AMF-SCM et ANMF-PFP amÈliorent les rËsultats. La figure ?? prËsente la carte Doppler-distance d'un des capteurs (simple transformation de Fourier des donnÈes acquises au cours du temps sur un seul capteur) des donnÈes contenues dans la case distance. 


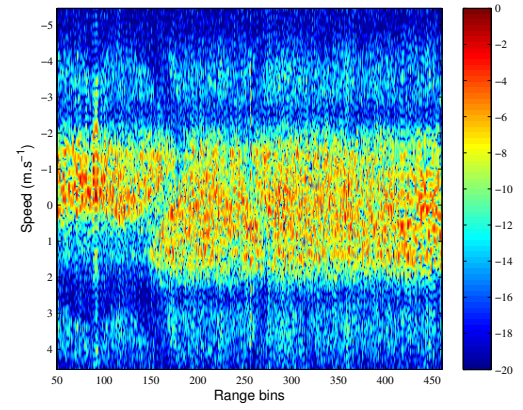

Figure 10. Carte Doppler-distance d'un des capteurs

\section{Conclusion}

Dans le cadre de la dÈtection radar et en particulier pour le STAP, l'estimation de la matrice de covariance du fouillis est gÈnÈralement une phase trËs importante et sa qualitÈ d'estimation influe fortement sur les performances de dËtection. Jusqu'ł trËs rÈcemment, presque tous les dÈveloppements thÈoriques Ètaient basÈs sur l'hypothËse gaussienne, que l'on peut quelquefois remettre en cause dans les situations opËrationnelles particuliËres (hËtËrogËnÈitÈ spatiale du fouillis, site de visÈe rasant, haute rÈsolution). De ce fait, de nouveaux modËles de fouillis, basÈs sur des processus gaussiens composÈs (SIRV) ont ÈtÈ proposÈs. Ils permettent de maniËre originale et efficace de prendre en compte l'hËtËrogĖnÈitÈ et la non-gaussianitÈ du fouillis. Toutes les techniques d'estimation et de dËtection peuvent Ítre alors revisitÈes. Dans le cadre gÈnÈrale de la dÈtection et de l'estimation dans le contexte SIRV, nous avons ainsi proposè de nouveaux dÈtecteurs basÈs sur de nouveaux estimateurs robustes de la matrice de covariance (matrice du point fixe) du fouillis. Ces techniques permettent d'amÈliorer fortement les performances des dÈtecteurs conventionnels basÈs sur l'hypothËse gaussienne.

Enfin, dans le cas du STAP latÈral, la matrice de covariance exhibe une structure particuliËre, la persymĖtrie, que nous avons exploitĖe pour encore amÈliorer ces performances et Ėgalement limiter le nombre de donnÈes secondaires nÈcessaire $\ddagger$ son estimation. Les rËsultats sur les signaux mis $\ddagger$ disposition dans le cadre du club STAP par la DGA MaÓtrise de l'Information, montrent l'intĖrÍt et la validitÈ de ces nouveaux dÈtecteurs.

\section{Remerciements}

Les auteurs remercient la DGA MaÓtrise de l'Information et THALES pour la mise $\ddagger$ disposition et l'exploitation de ses donnÈes. 


\section{Bibliographie}

Abramowitz, M., Stegun, I., C., (1972). Handbook of mathematical functions, New York, Dover.

Barnard T.J., Weiner D.D., (1996). Non-Gaussian clutter modeling with generalized spherically invariant random vectors, IEEE Trans. on Signal Processing, vol. 44, n 10, p. 2384-2390.

Bausson S., Pascal F., Forster P., Ovarlez J.P., Larzabal P., (2007). First and second order moments of the normalized sample covariance matrix of spherically invariant random vectors, IEEE Signal Processing Letters, vol. 14, $\mathrm{n}^{\circ}$ 6, p. 425-428.

Bidon S. (2011). Introduction au STAP. Partie II : ModËle des signaux et principe du filtrage, Revue Traitement du Signal.

Bidon S., MontÈcot M., Savy L., (2011). Introduction au STAP. Partie III : Les donnÈes du club STAP, Revue Traitement du Signal.

Bidon S., Besson O., Tourneret J.Y., (2011). SynthËse des traitements STAP pour la dÈtection en environnement hÈtÈrogËne, Revue Traitement du Signal.

Billingsley J.B., (1993). Ground clutter measurements for surface-sited radar, Technical Report 780, MIT, fÈvrier 1993.

Billingsley J.B., (2002). Low-Angle Radar Land Clutter. Measurements and Empirical Models, Norwitch, NY, William Andrew Publishing.

Bilodeau M., Brenner D., (1999). Theory of multivariate statistics, Springer Verlag.

Brennan L.E., Reed I.S., (1973). Theory of Adaptive Radar, IEEE Trans. on Aerospace and Electronic Systems, vol. 9, $\mathrm{n}^{\circ}$ 2, p. 237-252.

Burg J.P., Luenberger D.G., Wenger D.L., (1982). Estimation of Structured Covariance Matrices, Proc. of the IEEE, vol. 70, p. 963-974.

Cai L., Wang H., (1992). A persymmetric multiband GLR algorithm, IEEE Trans. on Aerospace and Electronic Systems, vol. 28, n 3, p. $806 \tilde{8} 816$.

Conte E., Lops M., Ricci G., (1995). Asymptotically Optimum Radar Detection in CompoundGaussian Clutter, IEEE Trans. on Aerospace and Electronic Systems, vol. 31, n 2, p. $617-$ 625.

Conte E., Ricci G., (1998). Sensitivity study of GLRT detection in compound-Gaussian clutter, IEEE Trans. on Aerospace and Electronic Systems, vol. 34, n 1, p. 308-316.

Conte E., Lops M., Ricci G., (1999). Incoherent radar detection in compound-Gaussian clutter, IEEE Trans. on Aerospace and Electronic Systems, vol. 35, $\mathrm{n}^{\circ}$ 3, p. 790-800.

Conte E., De Maio A., Galdi, C., (2000). Signal detection in compound-Gaussian noise : Neyman-Pearson and CFAR detectors, IEEE Trans. on Signal Processing, vol. 48, $\mathrm{n}^{\circ} 2$, pp.419-428.

Conte E., De Maio A., Ricci G., (2002). Recursive estimation of the covariance matrix of a compound-Gaussian process and its application to adaptive CFAR detection, IEEE Trans. on Signal Processing, vol. 50, $\mathrm{n}^{\circ}$ 8, p. 1908-1915.

Conte E., De Maio A., (2003). Exploiting persymmetry for CFAR detection in compoundGaussian Clutter, IEEE Trans. on Aerospace and Electronic Systems, vol. 39, n 2, p. 719724.

Conte E., De Maio A., (2004). Mitigation techniques for non-Gaussian sea clutter, IEEE Journal of Oceanic Engineering, vol. 29, n ${ }^{\circ}$ 2, p. 284-302. 
Fuhrmann D.R., (1991). Application of Toeplitz covariance estimation to adaptive beamforming and detection, IEEE Trans. on Signal Processing, vol. 39, p. 2194-2198.

Gini F., (1997). Sub-optimum coherent radar detection in a mixture of K-distributed and Gaussian clutter, IEE Proc. Radar, Sonar and Navigation, vol. 144, n 1, p. 39-48.

Gini F., Greco M.V., (2002). Covariance matrix estimation for CFAR detection in correlated heavy tailed clutter, Signal Processing, Special section on Signal Processing with Heavy Tailed Distributions, vol.. 82, n 12, p. 1847-1859.

Ginolhac G., Forster P., Ovarlez J.-P., Pascal F., (2011). STAP $\ddagger$ rang réduit, robuste et persymétrique, Revue Traitement du Signal.

Huber P.J., Ronchetti E. M., (2009), Robust statistics, John Wiley \& Sons Inc.

Jakeman E., (1980). On the Statistics of K-Distributed Noise, J. Phys. A : Math Gen., vol. 13, p. 31-48.

Jao J.K., (1984). Amplitude distribution of composite terrain radar clutter and the Kdistribution, IEEE Trans. on Antennas and Propagation, vol. 32, n 10, p. 1049-1062.

Jay E., (2002). DÈtection en environnement non-gaussien, ThËse de doctorat. UniversitÈ de Cergy Pontoise.

Kelker, D., Distribution theory of spherical distributions and a location-scale parameter generalization, (1970). Sankhya A, vol. 32, pp. 419-430.

Kelly E.J., (1986). An adaptive detection algorithm, IEEE Trans. on Aerospace and Electronic Systems, vol. 23, pp.115-127.

Korado V.A.,(1968). Optimum detection of signals with random parameters against the background of noise of unknown intensity under conditions of constant false alarm probability, Radio Engineering and Electronic Physics, vol. 13, p. 671-679.

Kraut S., Scharf L.L., (1999). The CFAR adaptive subspace detector is a scale-invariant GLRT, IEEE Trans. on Signal Processing, vol. 47, n ${ }^{\circ}$ 9, p. 2538-2541.

Kraut S., Scharf L.L., Mc Whorter L.T., (2001). Adaptive subspace detectors, IEEE Trans. on Signal Processing, vol. 49, $\mathrm{n}^{\circ}$ 1, p. 1-16.

Mahot M., Forster P., Ovarlez J.P., Pascal F., (2010). Robustness analysis of covariance matrix estimates, Actes EUSIPCO'10, Aalborg, Danemark, 23-27 ao 't 2010.

Maronna R. A., (1976). Robust M-estimators of multivariate location and scatter, Annals of Statistics, vol. 4, $\mathrm{n}^{\circ} 1$, pp. $51 \tilde{n} 67$.

MontÈcot M., Le Chevalier F., Savy L., (2011), Introduction au STAP. Partie I : Contexte radar et enjeu du filtrage, Revue Traitement du Signal.

Nitzberg R., Burke J.R., (1980). Application of maximum likelihood estimation of persymmetric covariance matrices to adaptive detection, IEEE Trans. on Aerospace and Electronic Systems, vol. 25, p. 124-127.

Pailloux, G., Forster P., Ovarlez J.P., Pascal F., (2008). On persymmetric covariance matrices in adaptive detection, Actes IEEE-ICASSP'08, Las Vegas, USA, p. 2305-2308.

Pailloux G., Ovarlez J.P., Pascal F., Forster P., (2008). A SIRV-CFAR adaptive detector exploiting persymmetric clutter covariance structure, Actes IEEE Radar Conference, Rome, Italie, p. $1139-1144$.

Pailloux G., Pascal F., Ovarlez J.P., Forster P., (2009). Comparison of various detection schemes for STAP radar based on experimental data, Actes IEEE-Radar'09, Bordeaux, France, 12-16 octobre 2009. 
Pailloux G., (2010). Estimation structurÈe de la covariance du bruit en dÈtection adaptative, ThËse de doctorat. UniversitÈ de Paris 10.

Pailloux G., Forster P., Ovarlez J.P., Pascal F., (2011). Persymmetric adaptive radar detectors, IEEE Trans. on Aerospace and Electronic Systems, $\ddagger$ paraÓtre.

Pascal F., Ovarlez J.P., Forster P. et Larzabal P., (2004). Radar detection In compound-Gaussian clutter, Actes IEEE Radar'04, Toulouse, France, 18-22 octobre 2004.

Pascal F., Ovarlez J.P., Forster P. et Larzabal P., (2004). Constant false alarm rate detection in spherically invariant random processes, Actes EUSIPCO'04, Vienne, Autriche, 6-10 septembre 2004, p. 2143-2146.

Pascal F., Ovarlez J.P., Forster P. et Larzabal P., (2005). Theoretical analysis of an improved covariance matrix estimator in non-Gaussian noise, Actes IEEE-ICASSP'05, Philadelphie, USA, 18-23 mars 205.

Pascal F., (2006). DÈtection et estimation en environnement non-gaussien", ThËse de doctorat. UniversitÈ de Paris 10.

Pascal F., Ovarlez J.P., Forster P., Larzabal P., (2006). On a SIRV-CFAR detector with radar experimentations in impulsive noise, Actes EUSIPCO'06, Florence, Italie, 4-8 septembre 2006.

Pascal F., Chitour Y., Ovarlez J.P., Forster P., Larzabal P., (2008). Covariance structure maximum likelihood estimates in compound Gaussian noise : existence and algorithm analysis, IEEE Trans-SP, vol. 56, n ${ }^{\circ} 1$, p. 34-48.

Pascal F., Forster P., Ovarlez J.P., Larzabal P., (2008). Performance analysis of covariance matrix estimates in an impulsive noise, IEEE Trans. on Signal Processing, vol. 56, $\mathrm{n}^{\circ}$ 6, p. 22062217.

Picinbono B., Vezzosi G., (1970). DÈtection d'un signal certain dans un bruit non stationnaire et non-gaussien, Annales des TÈlÈcommunications, vol. 25, p. 433-439.

Rangaswamy M., Weiner D.D., Öztürk A., (1993). Non-Gaussian random vector identification using spherically invariant random processes", IEEE Trans. on Aerospace and Electronic Systems, vol. 29, n ${ }^{\circ}$ 1, p. 111-123.

Reed I.S., Mallet J.D., Brennan L.E, (1974). Rapid convergence rate in adaptive arrays, IEEE Trans. on Aerospace and Electronic Systems, vol. 10, $\mathrm{n}^{\circ}$ 10, p .853-863.

Robert C.P., (1994). The Bayesian Choice : a Decision-Theoretic Motivation, New York, Springer-Verlag.

Robey F.C., Fuhrmann D.R., Kelly E.J., Nitzberg R., (1992). A CFAR adaptive matched filter detector, IEEE Trans. on Aerospace and Electronic Systems, vol. 28, n ${ }^{\circ}$ 1, p. 208-216.

Sangston K.J., Gini F., Greco M.V., Farina A., (1999). Structures for radar detection in compound gaussian clutter, IEEE Trans. on Aerospace and Electronic Systems, vol. 35, n 2, p. $445-458$.

Scharf L.L., Lytle D.W., (1971). Signal detection in Gaussian noise of unknown level : an invariance application, IEEE Trans. on Information Theory, vol. 17, p. 404-411.

Scharf L.L., Friedlander B., (1994). Matched subspace detectors, IEEE Trans. on Signal Processing, vol. $42, \mathrm{n}^{\circ} 8$, p. 2146-2157.

Tyler D., (1983). Robustness and efficiency properties of scatter matrices, Biometrika, vol. 70, $\mathrm{n}^{\mathrm{o}} 2, \mathrm{p} .411$. 
Vasile G., Ovarlez J.P., Pascal F., Tison C., (2010). Coherency matrix estimation of heterogeneous clutter in high resolution polarimetric SAR images, IEEE Trans. on Geoscience and Remote Sensing, vol. 48, n 4, p. 1809-1826.

Yao K., (1973). A representation theorem and its applications to spherically invariant random processes, IEEE Trans. on Information Theory, vol. 19, $\mathrm{n}^{\circ}$ 5, p. 600-608. 